Stefan M. Maul

\title{
Ein altorientalischer Pferdesegen - Seuchenprophylaxe in der assyrischen Armee
}

\author{
Der verehrten Jubilarin \\ zum Zeichen der Hochachtung und Freundschaft
}

\begin{abstract}
Sumerisch-akkadische Beschwörungen „um den Pferdehof zu reinigen“ sind auf der mittelassyrischen Tafel VAT 10035 (KAR 91) aus einer Palastbibliothek in Assur und den neuassyrischen Textvertretern Sm 1708 (IVR2 Pl. 18* Nr. 6), ND 4405/47 (CTN 4, 101) und VAT 11019 überliefert. Dieser Beitrag liefert die Edition dieser Beschwörungen und der entsprechenden Ritualanweisungen, die im Kontext der Bedeutung von Pferden für die assyrische Armee zu sehen sind.
\end{abstract}

Während seit alters her Herden von Wildeseln die Halbwüsten bevölkerten, welche die Flußoase von Euphrat und Tigris umgeben, war das Wildpferd in den Ebenen des Zweistromlandes nie heimisch. ${ }^{1}$ In der mesopotamischen Hochkultur kannte man es freilich schon früh, da die umliegenden Gebirgsregionen von Zagros und Taurus zu seinem natürlichen Lebensraum gehören. Die Sumerer nannten es dementsprechend „Bergesel (a ň e-kur$\mathrm{ra}$ )“. Als domestiziertes Haustier gewann das Pferd in Mesopotamien von der Mitte des dritten vorchristlichen Jahrtausends an nach und nach an Bedeutung. Obgleich es sich als Zugtier für die Wagen von Fürsten, Königen und Göttern bald großer Beliebtheit erfreute, blieb es so unerschwinglich, daß es im zivilen Bereich in Mesopotamien den Hausesel als Zug-, Last- und Reittier nie verdrängen konnte.

\section{Militärpferde für Assyrien}

Die Erfindung eines leichten zweirädrigen, von zwei, drei oder vier Pferden gezogenen Kampfwagens veränderte um die Mitte des 2. Jt. v. Chr. die altorientalischen Formen

$1 \mathrm{Zu}$ Pferden in Mesopotamien siehe den grundlegenden Beitrag von M. Weszeli, Pferd. A. I. In Mesopotamien, in: RlA 10/5-6 (2004) 469-481 mit weiterführender Literatur. Siehe ferner R. H. Meadow/ H.-P. Uerpmann (Hg.), Equids in the ancient world. TAVO Bh. A 19/1 (Wiesbaden 1986) und TAVO Bh. A 19/2 (Wiesbaden 1991). Zu Pferden im alten Ägypten siehe L. A. Heidorn, The horses of Kush, JNES 56 (1997) 105-114 und ferner O. Loretz, Das pharaonische Wagengespann mit Stute des Canticums (1,9-11) in hippologisch-militärgeschichtlicher Sicht, UF 36 (2004) 205-234. Zu Pferden im alten Anatolien siehe Th. van den Hout, Pferd (und weitere Equiden). A. II. In Anatolien, in: RlA 10/5-6 (2004) 482-490.

der Kriegsführung von Grund auf. ${ }^{2}$ Denn bei geeignetem Gelände erwiesen sich Schnelligkeit und Wendigkeit dieses neuen, in Nordsyrien entwickelten Kriegsgeräts oft als entscheidend für das Schlachtgeschehen. Die Armeen der altorientalischen Mächte verlangten daher nun nicht nur nach den leichten Streitwagen mit zwei Speichenrädern, sondern auch mehr als je zuvor ${ }^{3}$ nach den zugehörigen Pferden, die sich - wenn man sie nur entsprechend abrichtete - in der Schlacht weit besser bewährten als die störrischen Hausesel.

Zwar wurde bald auch im Zweistromland Pferdezucht betrieben, ${ }^{4}$ aber der Bedarf an Nachschub, den nicht zuletzt die Armee des stark expandierenden Assyrien brauchte, ließ sich damit nicht decken. Ein großer Teil der benötigten Tiere mußte aus den umliegenden Gebirgsregionen des Taurus, des ostanatolischen Hochlandes und des Zagros, wo Pferde wild lebten, aber auch in ihrem natürlichen Lebensraum gezüchtet wurden, herbeigeschafft werden, sei es durch Handel, als Geschenke oder als Beutegut.

Ganze Feldzüge wurden ausgerichtet, um die assyrische Armee in den Besitz der kostbaren Tiere zu bringen. Gelang es, ein Gebiet, in dem Pferde heimisch waren, zu unterwerfen, hatten die Besiegten eine große Zahl der Tiere als Tribut zu erbringen. ${ }^{5}$ Der mittelassyrische König

2 Siehe P. R. S. Moorey, The emergence of the light, horse-drawn chariot in the Near East, c. 2000-1500 B.C., World Archaeology 18 (1986) 196-215.

$3 \mathrm{Zu}$ der Rolle des Pferdes in den Armeen der altbabylonischen Zeit siehe J. Eidem, RA 85 (1991) 131-134.

4 Siehe Weszeli, RIA 10, 472-473 mit weiterführender Literatur. 5 Neben Weszeli, RIA 10, 472 siehe die Zusammenstellung von Abbildungen auf assyrischen Monumenten, auf denen Pferde als Tributgabe abhängiger Völkerschaften gezeigt werden, in: J. Bär, Der assyrische Tribut und seine Darstellung. AOAT 243 (Kevelaer/Neukirchen-Vluyn 1996) 238. 
Tiglatpileser I. (1114-1076 v. Chr.) etwa erbeutete, seinen eigenen Berichten zufolge, in den Gebirgszügen des Taurus südöstlich von Van ganze Pferdeherden und verlangte von den Einheimischen, jährlich 1200 Tiere nach Assyrien zu liefern. ${ }^{6}$ Auch in die Gebirgszüge des Zagros unternahmen die Assyrer regelmäßige Tributexpeditionen, um den Tierbestand ihrer Armee zu erweitern. In Mesopotamien galt darüber hinaus noch im ersten vorchristlichen Jahrtausend das „Gebirge Harsamna“, eine zentralanatolische Region, die schon in altbabylonischer Zeit für ihre Pferde und die Pferdezucht zu Berühmtheit gelangt war,7 als der sagenhafte „Berg der Pferde.“"

Nach Assyrien gelangt, mußten die Pferde an ihr neues Umfeld gewöhnt und auf das Kampfgeschehen, in dem sie Einsatz finden sollten, sorgfältig vorbereitet werden. Die professionelle Dressur der Tiere war unabdingbare Voraussetzung für eine militärische Überlegenheit der Streitwagentruppen. In der Schlacht sollten die Pferde schließlich, ohne zu scheuen, jedem Befehl des Wagenlenkers gehorchen und dabei ebenso schnell wie wendig sein. Es liegt auf der Hand, daß das Wissen um die Finessen der militärischen Pferdedressur für alle vorderasiatischen Großmächte der Zeit hohe sicherheitspolitische Bedeutung besaß. In der assyrischen Hauptstadt und Königsresidenz Assur erachtete man es im ausgehenden zweiten vorchristlichen Jahrtausend für so wesentlich, daß es schriftlich fixiert und sogar - ganz so wie königliche Erlasse - auf sorgsam gebrannten Tontafeln festgehalten wurde. In diesem für das Militär bestimmten, in assyrischer Sprache verfaßten Schrifttum ${ }^{9}$ finden sich Anweisungen, wie die Wagenpferde zu pflegen und für ihren Einsatz vorzubereiten sind. Die in eigenen Stallungen untergebrachten Tiere hatten vor den Streitwagen gespannt jeweils auf ganz unterschiedlichem Gelände verschiedene Gangarten, Drehungen und Wendungen zu erlernen und immer wieder zu üben. Die assyrischen Richtlinien für Pflege und Training der Militärpferde dürften sich an vergleichbaren, ein wenig älteren hethitischen Schriften ${ }^{10}$ orientieren und das ursprünglich wohl in Anatolien entstandene Fachwissen weiterführen.

6 A. K. Grayson, RIMA 2 (Toronto 1991) 22, Text 1 v 5-21.

7 Siehe D. Charpin, Die Beziehungen zwischen Anatolien und der syro-mesopotamischen Welt in der altbabylonischen Zeit, in: G. Wilhelm (Hg.), Hattuša - Boğazköy. CDOG 6 (Wiesbaden 2008) 95-107, besonders 95 mit Anm. 1 und 105 mit Anm. 44.

8 Siehe E. Reiner, Lipšur-litanies, JNES 15 (1956) 129-149, besonders 134, Z. 43.

9 E. Ebeling, Bruchstücke einer mittelassyrischen Vorschriftensammlung für die Akklimatisierung und Trainierung von Wagenpferden (Berlin 1951).

10 CTH 284-287, siehe dazu http://www.hethport.uni-wuerzburg.de/CTH/ mit weiterführender Literatur.
Im ersten vorchristlichen Jahrtausend zwangen die nicht immer glücklichen Erfahrungen mit nomadisierenden Reiterkriegerverbänden die Assyrer zum Aufbau einer selbständigen Kavallerie, deren Beweglichkeit sich rasch als äußerst vorteilhaft erwies. Erneut stieg der Bedarf an Pferden erheblich an. Die berittenen Soldaten, die zumeist Pfeil und Bogen, Speer, einen kleinen Schild und ein Kurzschwert führten, lösten nach und nach die Streitwageneinheiten ab, so daß der Streitwagen im frühen 7. Jh. v. Chr. nur noch Zeremonialcharakter besaß.

Um die Wehrbereitschaft sicherzustellen, wurden auch die Pferdebestände der Armee regelmäßig gemustert. Hiervon zeugen keilschriftliche Musterungsprotokolle, die in fast allen assyrischen königlichen Residenzen gefunden wurden und allesamt aus der neuassyrischen Zeit stammen. ${ }^{11}$ Die ausführlichsten Berichte über die $\mathrm{Mu}$ sterung von Pferden fanden sich in den Ruinen des Zeughauspalastes der frühneuassyrischen Königsresidenz Kalhu. ${ }^{12}$ Sie wurden im späten 8 . Jh. v. Chr. verfaßt. In Listen hatten Fachleute für die Heeresleitung zusammengestellt, zu welcher Verwendung die etwa 3000 unter der Verantwortung jeweils eines Offiziers stehenden Tiere geeignet erschienen, ob sie bereits auf einem Feldzug eingesetzt worden oder „im Lande“ geblieben waren, ob sie eines weiteren Trainings bedürfen und ob man sie für einen Einsatz bei einem neuen Feldzug verwenden könne.

\section{Hege und Pflege der Pferde in den assyrischen Militärlagern}

Es versteht sich von selbst, daß den großen Pferdehöfen des assyrischen Heeres auch Veterinäre zur Verfügung standen, ${ }^{13}$ denn eine Seuche, die unter Militärpferden aus-

11 Im sog. Alten Palast in Assur wurde ein kleines Archiv mit Protokollen über die Musterung von Pferden gefunden (KAV 31-38 und 131-132; vgl. O. Pedersén, Archives and libraries in the city of Assur I [Uppsala 1985] 30 Anm. 7). Aus den königlichen Archiven zu Ninive stammen verwandte Texte (F. M. Fales/J. N. Postgate, Imperial administrative records II. SAA 11 [Helsinki 1995] xxiv-xxvi und passim; ferner S. W. Cole/P. Machinist, Letters from priests to the kings Esarhaddon and Assurbanipal. SAA 13 [Helsinki 1998] xvii-xviii sowie Texte Nr. 82 ff.).

12 S. Dalley/J. N. Postgate, The tablets from Fort Shalmaneser. CTN 3 (London 1984) 17-22, 27-47 und Texte Nr. 85 und 98-118.

13 Vgl. hierzu grundlegend M. Stol, Das Pferd und seine Krankheiten im alten Babylonien, in: O. Loretz (Hg.), Hippologia Ugaritica. Das Pferd in Kultur, Wirtschaft, Kriegführung und Hippiatrie Ugarits Pferd, Esel und Kamel in biblischen Texten. AOAT 386 (Münster 2011) 363-402. 
bricht, kann von einem Tag auf den anderen die Wehrtüchtigkeit einer ganzen Armee zunichte machen, wenn deren Schlagkraft zu einem großen Teil auf Streitwagenkompanien oder der Kavallerie beruht. Das Fachwissen der Pferdedoktoren wurde daher so hoch geachtet, daß es in die schriftliche Überlieferung einging. In einem sehr umfangreichen, in Assur gefundenen Tontafelverzeichnis aus dem 7. Jh. v. Chr., in dem zahlreiche humanmedizinische Traktate aufgelistet sind, ist ganz am Ende auch eine Tontafel genannt, die der Pferdeheilkunde gewidmet war. ${ }^{14}$ Leider blieb sie uns bis heute unbekannt. In einem humanmedizinischen keilschriftlichen Kompendium des 7. vorchristlichen Jahrhunderts, in dem Heilmittel gegen verschiedene innere Krankheiten, Augen- und Zahnleiden sowie weitere Beschwerden zusammengestellt sind, die man auf das Wirken eines Totengeistes zurückführte, finden sich immerhin auch zwei Rezepte, welche die Heilung der gefürchteten Koliken (kīs libbi) beim Pferd versprechen. ${ }^{15}$ Im ersten Fall empfiehlt der Arzt eine Lösung von acht Pflanzen in gekeltertem Wein, die in die linke Nüster des erkrankten Tieres zu gießen sei. ${ }^{16}$ Im zweiten beschreibt er die Herstellung und Verabreichung eines Einlaufs, der aus insgesamt 23 Pflanzen hergestellt wurde. Die Pflanzen sollten über Nacht unter dem Einfluß des ,Ziegensterns' in Bier eingeweicht, gekocht und der Sud gefiltert werden. Mit weiteren Ingredienzien, einem Liter Honig und einem Liter Öl wurde das Gemisch in einen Trinkschlauch gefüllt und anschließend durch ein Rohr als Einlauf verabreicht. Pro Pferd sollten dabei vier Liter gekochtes Bier verwendet werden. ${ }^{17}$ Weitere vergleichbare, aber bedeutend ältere Schriften zur Pferdeheilkunde stammen aus Ugarit. ${ }^{18}$ Sie wurden im 13. Jh. v. Chr. in der ugaritischen Keilalphabetschrift aufgezeichnet.

Der machtpolitisch keineswegs unwichtigen Aufgabe, die Militärpferde, die in der Hauptstadt und in den königlichen Residenzen Assyriens stationiert waren, vor Krank-

14 Bruchstücke des Verzeichnisses sind veröffentlicht in: G. Beckman/B. R. Foster, Assyrian scholarly texts in the Yale Babylonian Collection, in: E. Leichty [u.a.] (Hg.), A scientific humanist. Studies in memory of Abraham Sachs (Philadelphia 1988) 1-26, als Texte Nr. 9a-d.

15 Der in Assur in den Ruinen des sog. Hauses des, Beschwörungspriesters' gefundene neuassyrische Text wird heute in den Staatlichen Archäologischen Museen zu Istanbul unter der Signatur A 198 aufbewahrt. Eine Keilschriftautographie veröffentlichte F. Köcher in BAM als Text Nr. 159. Zu den pferdeheilkundlichen Passagen des Textes siehe Stol, AOAT 386, 387-399 sowie C. Cohen, JANES 15 (1983) 1-12. 16 Das Rezept findet sich in BAM 159 v 33-36.

17 Das Rezept findet sich in BAM 159 v 37-47.

18 Siehe D. Pardee, Les textes hippiatriques, Ras Shamra-Ougarit 2 (Paris 1985) und AOAT 386, $179 \mathrm{ff}$. heiten und Seuchen zu schützen, versuchte man mit allen zur Verfügung stehenden Mitteln gerecht zu werden. Daher kümmerten sich nicht allein Tierpfleger und Veterinäre um den Zustand der kostbaren und für die Sicherheit des Landes so wichtigen Pferde. Stets unterstanden die königlichen Stallungen und die dort untergebrachten Tiere auch der Sorge von „Beschwörern“ (āšipu, mašmaššu), die mit ihrem esoterischen, auf die Götter selbst zurückgeführten Wissen in den Palästen für das Wohlergehen von Mensch und Tier zu sorgen hatten.

Aus den königlichen Bibliotheken zu Ninive ist die im 7. Jh. v. Chr. niedergeschriebene, recht ausführliche Beschreibung eines Verfahrens bekannt, das empfohlen wurde, „um Kopfkrankheit, Seuche und ,Pest“ an die Pferde und die Truppen des Königs nicht herankommen zu lassen. “19 Der König selbst hatte hierfür an feierlichen Opferzeremonien teilzunehmen und Bitt- und Bußgebete in sumerischer Sprache zu rezitieren. Erst dann wurde aus zahlreichen Ingredienzien eine Paste gekocht und in Bier und ,Weihwasser (agubbû) ' gelöst. „Die (im) Feldlager (befindlichen) Pferde“, heißt es dann in der an den Beschwörer gerichteten Anweisung, „hältst du fest (und) besprengst (mit der zuvor hergestellten Flüssigkeit) das Feldlager." ${ }^{20}$ Mit einer aus 15 Ingredienzien bestehenden Salbe, welche u.a. Blut eines Lamms, eines Vogels, eines Ochsen und eines weißen Pferdes enthielt, sollten im folgenden nicht nur Türschwellen und Toreingänge des Heereslagers bestrichen, sondern auch Lederbeutelchen gefüllt werden, die man den Pferden zu ihrem Schutz als Amulett um den Hals band.

Bei der Herstellung von Pferdegeschirr und Pferdeschmuck wurden - wohl nach Anweisungen der Beschwörer - nicht selten Materialien verarbeitet, denen man eine schützende Wirkung zuschrieb. Eine Art Breccia, die die Assyrer turminabandû nannten, erachtete man als besonders wirksam, „um den Wagen des Königs und des Fürsten zu schützen, um die Pferde zu schützen, um in der Schlacht zu schützen, damit sich Bedrängnis in der Schlacht dem Menschen nicht nähere, damit ein übelwollender Gott sich dem Menschen nicht in den Weg stellt.“21

19 R. Caplice, Namburbi texts in the British Museum IV, Or. 39 (1970) 118-124 und Tab. III-IV (82-3-23, 1). Zu der Tafel siehe auch S. M. Maul, Zukunftsbewältigung. BaF 18 (Mainz 1994) 220 und I. Huber, Rituale der Seuchen- und Schadensabwehr im Vorderen Orient und Griechenland. Formen kollektiver Krisenbewältigung in der Antike (Stuttgart 2005) 24-30.

20 Ebd. Z. 53.

21 A. Schuster-Brandis, Steine als Schutz- und Heilmittel. Untersuchungen zu ihrer Verwendung in der Beschwörungskunst Mesopotamiens im 1. Jt. v. Chr. AOAT 46 (Münster 2008) 354-355, Z. 9-12. 
Wohl im Glauben an die Kraft der Steine wurden etwa steinerne Aufstecker für Trensenknebel ${ }^{22}$ gefertigt, die man den in die Schlacht ziehenden Pferden anlegte. Der neuassyrische König Sanherib (705-681 v. Chr.) ließ sogar, wie wir aus einer seiner Inschriften wissen, vor den Pferdekrippen der in Ninive neu errichteten königlichen Stallungen Splitter von Amulettsteinen im Boden vergraben, damit deren stärkende Wirkung den Pferden dauerhaft zuteil würde. ${ }^{23}$

Auch das hippiatrische Fachwissen der Beschwörer lag in schriftlicher Form vor. Es war in einem vermutlich längeren Traktat zusammengestellt, von dem wir lediglich den Titel kennen. Dieser ist in einem im 7. Jh. v. Chr. niedergeschriebenen Katalog genannt, in dem die wichtigsten Schriften, „welche für die Lehre und das Studium (eines āšipu bzw. eines mašmaššu) verbindlich sind," ${ }^{24}$ namentlich aufgeführt wurden. Das von den Beschwörern mit dem Titel „Um den Rinder-, Schaf- und Pferdehof reinzuhalten" ${ }^{25}$ zitierte Werk enthielt Beschreibungen von Verfahren, die in Viehhürden, Gestüten und Heerlagern das Ausbrechen von Seuchen unter den Tieren verhindern sollten. ${ }^{26}$

\section{Ein altorientalischer Pferdesegen}

\section{Die Textvertreter}

Es ist nicht unwahrscheinlich, daß eine Tafel, die im Herbst 1913 im Schutt des Alten Palastes in Assur gefun-

22 Siehe B. Muhle, Steinerne Trensenknebelaufstecker in Assur. Elemente reiternomadischen Zaumzeugs, MDOG 137 (2005) 91-97.

23 J. MacGinnis, Some inscribed horse troughs of Sennacherib, Iraq 51 (1989) 187-192.

24 Die jüngste Bearbeitung des sog. ,Leitfadens der Beschwörungskunst‘ legte M. J. Geller vor, in: A. R. George/I. L. Finkel (Hg.), Wisdom, gods and literature. Studies in assyriology in honour of W. G. Lambert (Winona Lake, Ind. 2000) 242-254, Text E; siehe auch C. Jean, La magie néo-assyrienne en contexte. Recherches sur le métier d'exorciste et le concept d'āšipūtu. SAAS 17 (Helsinki 2006) 62-82 und die Übersetzung von K. Hecker, TUAT NF 4 (Gütersloh 2008) 76-79. Einen weiteren Textvertreter aus dem seleukidenzeitlichen Uruk legte E. von Weiher, SpTU 5 (Mainz 1998) 20-21 Text Nr. 231 vor. 25 tùr áb-gu $u_{4}$-hi-a $u_{\text {us }}$-udu-hri-a anše-kur-ra sikile-dè (siehe Geller, Fs. Lambert, 250, Z. 24 und von Weiher, SpTU 5, 231: 24).

26 Es ist nicht auszuschließen, daß die oben besprochene, in Ninive gefundene Tafel 82-3-23, 1 (siehe Anm. 19) Teil dieses Traktates war. $\mathrm{Zu}$ Beschreibungen von Verfahren der Seuchenprophylaxe im Heerlager, die die Hethiter schon im letzten Drittel des 2. Jt. überlieferten, siehe z.B. СTH 394, 424, 425 (siehe dazu http://www.hethport.uniwuerzburg.de/CTH/ mit weiterführender Literatur). den wurde und zu den wenigen Resten einer mittelassyrischen Palastbibliothek ${ }^{27}$ gehört, als Teil dieses Werkes betrachtet wurde. Die heute im Vorderasiatischen Museum zu Berlin aufbewahrte Tafel mit der Signatur VAT 10035 (Fundnr. Ass 22790 a; hier Textvertreter A ${ }^{28}$ ) enthält die detaillierte Beschreibung eines Pferdesegens, mit dem man „den Pferdehof reinzuhalten“ und die kostbaren Tiere vor der Gefahr von Krankheit und Seuche zu bewahren suchte.

Für wie wichtig man das in dieser Tafel beschriebene Verfahren hielt, läßt sich zum einen daran ermessen, daß in der späten mittelassyrischen Zeit ${ }^{29}$ der „Beschwörer des Königs“ diese Tafel eigenhändig von „einem alten Schriftstück aus dem Land Akkad“ abschrieb und dafür sorgte, daß sie im Königspalast zur Verfügung stand. Zum anderen zeigt sich die große Bedeutung, die man dem alten mesopotamischen Pferdesegen zusprach, darin, daß auch im ersten vorchristlichen Jahrtausend in allen wichtigen Zentren des assyrischen Reiches Abschriften dieses Textes bereit gehalten wurden. Wir können nämlich mittlerweile nicht nur einen wohl in der sehr frühen neuassyrischen Zeit (?) geschriebenen weiteren Textvertreter aus Assur nachweisen (VAT 11019; hier Textvertreter D), ${ }^{30}$ sondern auch eine jüngere, wohl im 8. Jh. v. Chr. entstandene Abschrift beibringen, die im Nabû-Tempel von Kalhu aufbe-

$27 \mathrm{Zu}$ dieser Bibliothek s. S. M. Maul, Die Reste einer mittelassyrischen Beschwörerbibliothek aus dem Königspalast zu Assur, in: W. Sallaberger [u.a.] (Hg.), Literatur, Politik und Recht in Mesopotamien. Festschrift für Claus Wilcke (Wiesbaden 2003) 181-194 und Pedersén, Archives and libraries I, 29-31 (M1. Small library in the Old Palace). Zum Fundort der Tafel s.a. F. Pedde/S. Lundström, Der Alte Palast in Assur. Architektur und Baugeschichte. WVDOG 120 (Wiesbaden 2008) 116 Nr. 1115.

28 Keilschriftautographie: Ebeling, KAR I (Leipzig 1915-1919) Text Nr. 91 und unten S. 32-33..

29 Die Zeichenformen, die das Schriftbild von VAT 10035 prägen, sprechen dafür, daß die Tafel im Zeitalter des assyrischen Königs Tiglatpilesers I. (12.-11. Jh. v. Chr.) geschrieben wurde. Der Schreiber, der königliche Beschwörer namens Rībātu, ist uns auch aus einer mittelassyrischen Urkunde bekannt (hierzu siehe den Kommentar zu dem Kolophon von VAT 10035), die in dem Jahr ausgestellt wurde, als Mutakkil-Aššur Eponym war. Nach H. Freydank „kommen das 13. bis 11. Jahrhundert für das Eponymat des M. in Frage“ (H. Freydank, Beiträge zur mittelassyrischen Chronologie und Geschichte [Berlin 1991] 154).

30 Die Fundnummer dieses bislang gänzlich unveröffentlichten, nun als Duplikat zu VAT 10035 identifizierten Tafelbruchstückes ging leider verloren, so daß über die Fundstelle und damit über die Bibliothekszugehörigkeit des Stückes keine Aussagen mehr getroffen werden können. Möglicherweise stammt es aus einer Tafelsammlung, die im Assur-Tempel aufbewahrt wurde. Es ist nicht völlig auszuschließen, daß VAT 11019 statt in der sehr frühen neuassyrischen in der ganz späten mittelassyrischen Zeit geschrieben wurde. 
wahrt wurde (ND 4405/47; hier Textvertreter C), ${ }^{31}$ sowie ein weiteres, schon seit langem bekanntes Manuskript, das in der Zeit des Assurbanipal (669-631? v. Chr.) für die königlichen Bibliotheken von Ninive angefertigt wurde (Sm 1708; hier Textvertreter B). ${ }^{32}$ Der in Assur gefundene Textvertreter D enthält auf der Tafelvorderseite (Abb. 7) wenige Reste einer weiteren Ritualbeschreibung, in deren Mittelpunkt ebenfalls das Wohl von Pferden zu stehen scheint. Die Passage ist leider so schlecht erhalten, daß sie noch nicht hinreichend gedeutet werden kann.

\section{Sprachstand und Alter}

Einen Hinweis darauf, daß der im wesentlichen durch VAT 10035 (Textvertreter A) bekannt werdende Text tatsächlich, so wie im Kolophon vermerkt (A, Rs. 25-26), auf eine erheblich ältere Vorlage zurückgeht, mag man darin erkennen, daß in VAT 10035 und damit auch in der zugehörigen Vorlage sumerische Passagen noch ohne jegliche akkadische Übersetzung überliefert wurden. Im ausgehenden 2. Jt. v. Chr., also in jener Zeit, als der königliche Beschwörer Rībātu die Tafel VAT 10035 anfertigte, war man schon mehr und mehr dazu übergegangen, sumerische Texte regelmäßig ins Akkadische zu übertragen und die Übersetzungen - sei es in einer separaten Kolumne oder so wie in den Textvertretern B, C und D, die wohl allesamt aus dem ersten vorchristlichen Jahrtausend stammen, interlinear - hinzuzufügen. In VAT 10035 kommt auch der Sprachstand des Sumerischen der von uns als ,klassisch empfundenen sumerischen Literatursprache der frühen altbabylonischen Zeit so nahe, daß der Eindruck nicht von der Hand zu weisen ist, die sumerischen Passagen unseres Textes seien bereits in jener Zeit oder gar noch früher entstanden. In diesem Fall hätten die dicenda des ,Pferdesegens ' ihre literarische Form bereits erhalten, bevor das domestizierte Pferd in Mesopotamien zu namhafter Bedeutung gelangt war. Auch wenn dies auf den ersten Blick abwegig erscheint, mag dem gleichwohl so gewesen sein. Denn in den sumerischen dicenda unseres Textes kann abgesehen von einem einzigen Beleg (Z. 31': a n š e-kur-ra), der möglicherweise einer späteren Angleichung geschuldet ist, keine einzige der dort ver-

31 Keilschriftautographie: D. J. Wiseman, CTN 4 (London 1996) Nr. 101 und unten S. 35. Zahlreiche Fehler zeigen, daß die Tafel wohl von einem noch nicht allzu weit fortgeschrittenen Schreiberlehrling (möglicherweise auswendig nach dem Memorieren einer Textvorlage) geschrieben wurde.

32 Keilschriftautographie: T. G. Pinches, IVR² (London 1891) Pl. 18*, Text Nr. 6 und unten S. 34. wendeten Equidenbezeichnungen (a n š e: Z. 2', 5', 6', 9'; dùr ùr: Z. 9'; anšedùr: Z. 10'; eme ${ }_{6} / /$ ème: Z. $8^{\prime}, 31^{\prime}$; anšê̂Á.ARHुUS: Z. 31'; anšek u n g a : Z. 31') auf das Pferd bezogen werden. Lediglich in den den dicenda (zweifelsohne vergleichsweise spät) hinzugefügten Unterschriften (Z. 28', 46') findet sich die sumerische Bezeichnung für das Pferd, a n š e-ku r-ra. Daher kann es als durchaus wahrscheinlich angesehen werden, daß unser ,Pferdesegen “ ursprünglich ein ,Eselssegen' gewesen ist und entstanden war, um die Bestände an domestizierten Eseln, denen - wie etwa die sog. Standarte von Ur eindrucksvoll vor Augen führt ${ }^{33}$ in den frühen Armeen des südlichen Mesopotamien eine gewichtige Rolle zukam, vor einer Seuchengefahr zu schützen.

Die in akkadischer Sprache gehaltenen Anweisungen des Textes (Z. 47'-61') weisen mit dem intensiven Gebrauch von KV- und VK-Zeichen ${ }^{34}$ und der Schreibung ${ }^{d}+$ EN.KI für den Namen des Weisheitsgottes (Z. 52' ${ }^{\prime 35}$ orthographische Konventionen auf, die in spätaltbabylonischer Zeit üblich waren. Dennoch haben die akkadischen Anweisungen ihre Gestalt letztlich in mittelassyrischer Zeit erhalten. Dies zeigt z.B. der in $t u$-še-taq (Z. 58') vorliegende Assyriasmus.

Die Überlieferung unseres Pferdesegens von der mittel- in die neuassyrische Zeit scheint im wesentlichen auf assyrischen Quellen zu beruhen. Anderenfalls würde man in dem Textvertreter aus der Assurbanipal-Bibliothek wohl nicht auf Assyriasmen wie êtiqma (statt bab. ìtiqma; Z. 11', B Rs. 6) und bēte (statt bab. bīti; Z. 15', B Rs. 14) treffen. Die jüngeren Textvertreter B, C, D dürften allesamt auf eine mittelassyrische Vorlage zurückgehen. Hierauf weisen der spärliche Umgang mit Logogrammen, Graphien wie z.B. be-e-te (B Rs. 14), ki-is-pa (C Rs. $6^{\prime}$ ), ku-up-ra (D Rs. $9^{\prime}$ ) sowie die konsequente Beibehaltung des Akkusativs auf - $a$, der in neuassyrischer Zeit schon weitgehend außer Gebrauch gekommen war. Der frühneuassyrische Textvertreter $\mathrm{D}^{36}$ weist ferner (ohne daß dies als Besonder-

33 Vgl. die Abbildung in: B. Hrouda (Hg.), Der alte Orient. Geschichte und Kultur des Alten Vorderasiens (München 1991) 334-335 sowie ferner 336-337.

34 Z.B. ku-up-ra (Z. 50'), ki-is-pa (Z. 54'), ta-ka-sa-ap (Z. 55') und tuka-sa-ma (Z. $\left.57^{\prime}\right)$.

35 In dem jüngeren Textvertreter B wird der Name des Weisheitsgottes, wie erwartet, ${ }^{\mathrm{d}} \hat{E}-a$ geschrieben (Z. 15', B Rs. 14). Laut R. Borger, ABZ 85 ist die Schreibung ${ }^{d}$ EN.KI nur in sumerischem Kontext anzutreffen. Dennoch findet sie sich, wenngleich sehr selten, in altbabylonischen akkadischen Manuskripten, so in der altbabylonischen Fassung des Atramhasis-Mythos (siehe CT 46 Nr. 1 i 16 und passim; in den späteren Duplikaten steht statt dessen d ${ }^{\mathrm{E}}-a$ ).

36 Siehe etwa die Zeichenformen von ú und GA mit vier statt drei stehenden Keilen sowie die Schreibung der „HI“-Gruppe in den Zeichen 
heit zu werten wäre) ebenso wie der im 8. Jh. v.Chr. niedergeschriebene Textvertreter $\mathrm{C}^{37}$ noch manche graphische Eigenheiten auf, die den mittelassyrischen Schreibkonventionen sehr nahestehen.

\section{Die Prozedur des Pferdesegens}

Dank der Duplikate aus der neuassyrischen Zeit kann der auf der stark beschädigten Tafel VAT 10035 niedergeschriebene Text hier erstmals nahezu vollständig rekonstruiert ${ }^{38}$ und das darin beschriebene Ritualgeschehen vorgestellt werden.

Zunächst kam dem Beschwörer die Aufgabe zu, ein Salböl nach einer Rezeptur herzustellen, die ohne Zweifel im Ruf stand, uralt zu sein $\left(\mathrm{Z} .47^{\prime}-51^{\prime}\right)$. Hierfür verwendete er verschiedene Pflanzen, die sich auch bei der Heilung von Menschen immer wieder bewährt hatten, zermahlenen Panzer? zweier Schildkrötenarten, zwei verschiedene Arten von vermutlich getrockneten Heuschrecken, kazīruWurm und weißen Skorpion. All dies wurde mit Fischtran, Schweinefett, Butter und pflanzlichem Öl, mit Bitumen, Asphalt und Gips zu einer Paste verarbeitet. Jedes einzelne Pferd sollte damit gesalbt und so mit Abwehrkräften ausgestattet werden (Z. 51').

Zwar fehlen immer noch grundlegende Untersuchungen, die uns verläßlich Auskunft darüber erteilen könnten, worin man die Wirkkraft der verschiedenen, für das Heilmittel verwendeten Ingredienzien sah. Es darf aber als nahezu sicher gelten, daß nach Sicht der mesopotamischen Heiler die Schildkrötenpanzer der Salbe eben die Schutzqualitäten verlieh, die der Panzer auch für die Kröte besaß, und der weiße Skorpion jene Wehrkraft, die dem lebenden Tier eignet. Auch dem kazìru-Wurm und den Heuschrecken dürften vergleichbare Qualitäten beigemessen worden sein, die man in der Salbe einzufangen suchte. ${ }^{39}$

Durch Opfer und Gebet herbeigerufen (Z. $\left.52^{\prime}-54^{\prime}\right)$ sollten insgesamt sieben Götter ihre schützende Hand über

IM, GI etc. mit insgesamt fünf statt vier kleinen Winkelhaken passim in Textvertreter D.

37 So z.B. die Zeichenform von BA (Textvertreter C Vs. 6', 7') und TI (Vs. 8').

38 Moderne Editionen der Textvertreter B, C und D liegen nicht vor (zu Textvertreter B [Sm 1708] siehe C. Fossey, La magie assyrienne [Paris 1902] 302-306). Eine nun stark veraltete Teilbearbeitung von Textvertreter A (KAR 91) legte A. Tsukimoto vor (A. Tsukimoto, Untersuchungen zur Totenpflege (kispum) im alten Mesopotamien. AOAT 216 [Kevelaer/Neukirchen-Vluyn 1985] 178-183).

$39 \mathrm{Zu}$ der Rolle von Asphalt und Gips siehe Maul, Zukunftsbewältigung, 99. die Pferde halten: die beiden Götter der Heil- und Beschwörungskunst, Enki und dessen Sohn Asalluhi, sowie Šamaš, der Gott des Rechtes und der Gerechtigkeit; 40 der persönliche Gott und die persönliche Göttin des Besitzers der Pferde; 41 schließlich die „Herrin-der-Steppe“ genannte Unterweltsgöttin und Schakkan, der Gott der in der Steppe lebenden wilden und der domestizierten Tiere.

Für die Mesopotamier hing Wohl und Wehe der zu schützenden Pferde in besonderer Weise von dem zuletzt genannten Götterpaar ab. Denn die „Herrin-der-Steppe“ (Bēlet-șêri), in der man auch die „Schreiberin der Unterwelt" sah, verkörpert jene Macht, die es in der Hand hat, die lebensfeindlichen Gewalten, welche die Steppe beherrschen, zurückzuhalten oder aber auf die Menschen loszulassen. Zu diesen Gewalten zählten für die Mesopotamier auch die als Dämonen gedachten Kräfte, die als „Todesboten“ (Z. 16') ,wie ein wütender Sturm durch die Steppe wirbeln“ (Z.1 und 16') und ,sich von der Steppe auf zum Viehhof machen“ können (Z. 3'), um Mensch und Tier üble Krankheiten zu bringen. ${ }^{42}$ Ohne das Wohlwollen der „Herrin-der-Steppe“ wäre ein nachhaltiger Schutz vor diesen Krankheiten ebensowenig zu erreichen wie ohne die Hilfe des „Helden der Steppe“ (Z. 33'), Schakkan, der über ,seine“ Tieren wacht.

Aus diesem Grund sieht das Verfahren des ,Pferdesegens' vor, den Hof, in dem die Tiere gehalten wurden (é-tùr anše-kur-ra: Z. 28',46'), dem besonderen Schutz dieser beiden Gottheiten zu unterstellen. Beide sollten - verkörpert in jeweils einer? Standarte, die wohl

40 Die Triade Ea-Šamaš-Asalluhi wurde in den Therapien der Beschwörungskunst immer dann angerufen, wenn mit den Mitteln, welche die Weisheitsgötter Enki/Ea und dessen Heiland-ähnlicher Sohn Asalluhi den Menschen in der Kunst der āšipūtu offenbart hatten, ein göttliches Urteil revidiert werden sollte. Hierzu siehe Maul, Zukunftsbewältigung, 60-71.

41 Als Besitzer der Pferde kommen hier neben dem König selbst der Kronprinz oder andere hochgestellte Personen in Frage, die wichtige Ämter im assyrischen Militär bekleideten. Die Anrufung der persönlichen Götter des Besitzers der Tiere ist für das Gelingen des Rituals unabdinglich, da die mesopotamischen Heiler in einer Erkrankung der Tiere ein göttliches Urteil erkannt hätten, das zur Maßregelung des Pferdehofbesitzers vollstreckt wird und ohne Einwilligung seiner persönlicher Götter nicht hätte zustande kommen können. Die Revision eines solchen Urteils wäre aus der Sicht der mesopotamischen Heiler ohne die nachhaltige Besänftigung dieser Götter nicht zu erreichen.

42 Die Vorstellung, daß aus dem lebensfeindlichen Raum, aus der Unterwelt, der Steppe und auch vom Himmel herab böse Gewalten hervorbrechen, um Tier und Mensch gemeinsam Schaden zuzufügen, findet sich immer wieder in der mesopotamischen Literatur der ,Beschwörungskunst'. Siehe z.B. B. Böck, Das Handbuch muššu’u „Einreibung“. Eine Serie sumerischer und akkadischer Beschwörungen aus dem 1. Jt. vor Chr. (Madrid 2007) 93-108; Tafel I. 
im Bereich der Zugänge zu dem Pferdehof aufgerichtet wurde (Z. 56', Textvertreter C) - die „reine, majestätische“ (Z. 27'), „heilige, erhabene Wohnung (der Tiere)“ (Z. 32',

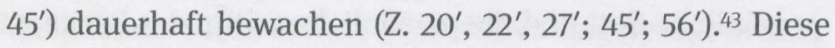
wurde mit gesegnetem Wasser besprengt (Z. $\left.38^{\prime}-41^{\prime}\right)^{44}$ und, um den bösen Mächten diesen Aufenthaltsort endgültig zu vergällen, mit Räuchergefäß und Fackel beschwenkt (Z. 23'-24', 42'-43').

Da man in dem in Z. $5^{\prime}-10^{\prime}$ und Z. $31^{\prime}-32^{\prime}$ beschriebenen, durch das Ritual fernzuhaltenden Leiden der Tiere auch das Wirken eines rachsüchtigen Totengeistes zu erkennen glaubte, der seine ewige Ruhe nicht finden konnte und der Familie des Pferdehofbesitzers entstammte, wurde auch diesem möglichen Störenfried gewissermaBen prophylaktisch zu seiner Besänftigung eine Speise bereitet, die man auch sonst den Verstorbenen als Totenopfer hinzustellen pflegte (Z. 54'-55'). 45

Waren Pferdehof und Tiere auf diese Weise vor dem unheilvollen Zugriff von außen geschützt, sollte auch alles unsichtbare Übel, das den Pferden anhaftete, aber noch nicht zu wahrnehmbaren Symptomen geführt hatte, beseitigt werden. Hierzu wurden auf dem Gelände des Pferdehofes große Hütten aus Rohr errichtet, in denen man eine Grube aushob, welche mit Holzbrettern wieder abgedeckt wurde, nachdem man darin ein lebendiges, gefesseltes weibliches Zicklein versenkt hatte. In einer langen Prozedur wurde daraufhin jedes einzelne Pferd durch eine der Rohrhütten und über die zugedeckte Grube mit

43 Der Pferdehof wird damit einem Verfahren unterzogen, das auch zum Schutz der menschlichen Behausung, z. B. in dem bīt mēseri genannten Ritual, zur Anwendung kam.

$44 \mathrm{Zu}$ solchem mit verschiedenen Ingredienzien aufbereiteten ,Weihwasser' siehe Maul, Zukunftsbewältigung, 41-46.

45 Hierzu siehe Tsukimoto, Totenpflege und J. Scurlock, Magico-medical means of treating ghost-induced illnesses in ancient Mesopotamia (Leiden 2006) passim. Es scheint, daß auch hier - so wie in vielen weiteren Texten der sog. Beschwörungskunst (āšipūtu) - keine klare Grenze gezogen wurde zwischen krankheitsbringenden Dämonen und Totengeistern, die der eigenen Familie angehören. der jungen Ziege geleitét (Z. 56'-58'). Darüber hinaus beschwenkte der Beschwörer zu Beginn der Nacht die zu schützenden Pferde mit einem lebenden Ziegenböckchen und wiederholte dies um Mitternacht und vor Sonnenaufgang. Drei Ziegenböckchen wurden hierfür ,verbraucht‘. Sie sollten ebenso wie die gebundene, in den Erdgruben liegenden Zicklein todbringendes Unheil von den Pferden nehmen und auf sich ziehen (Z. 58'-59'). 46

Im Morgengrauen wurden dann alle bösen Kräfte, die die Pferde noch umgeben könnten, durch den Lärm von Paukenschlägen und glocken- oder gongartigen Instrumenten vertrieben und die Tiere mit Blättern, Räucherwerk und ,Weihwasser` ein letztes Mal symbolisch gereinigt (Z. 60'-61'). Auf diese Weise sollten die Pferde vor allen drohenden Übeln gefeit sein. Die Zicklein, die gebunden in der Grube gelegen hatten, und die Böckchen, die man den Pferden entgegengeschwenkt hatte, wurden wohl, auch wenn davon in unserem Text nicht mehr die Rede ist, als ,Sündenböcke‘ davongejagt oder getötet. So wurde Leben für Leben gegeben, in der Hoffnung, daß höhere Mächte nun für die Gesundheit der Pferde sorgen würden.

Leider fehlen uns jegliche Angaben darüber, bei welcher Gelegenheit man den ,Pferdesegen' durchführte. So kann man nur vermuten, daß das Ritual jährlich an einem eigens dafür festgesetzten Datum stattfand, möglicherweise unter großer Anteilnahme von Soldaten und Bevölkerung, eingebettet in ein Fest.

46 Entsprechende Verfahren kamen auch zum Schutz des Menschen und seiner Behausung zur Anwendung. 


\section{Edition des Textes ${ }^{47}$}
$A=$ VAT 10035
Z. 1'-61'; Kolophon
Kopie: E. Ebeling, KAR I 161-162 Text Nr. 91 und unten Abb. 1-2, S. 32-33.
$\mathrm{B}=\mathrm{Sm} 1708$
Z. $1^{\prime}-22^{\prime}$
Kopie: T. G. Pinches, IVR ${ }^{2}$ Pl. $18^{\star}$ Text Nr. 6 und unten Abb. 3-4, S. 34.
$\mathrm{C}=\mathrm{ND} 4405 / 47$
Z. $9^{\prime}-17^{\prime} ; 49^{\prime}-59^{\prime}$
Kopie: D. J. Wiseman, CTN 4 Pl. 61 Text Nr. 101 und unten Abb. 5-6, S. 35.
$\mathrm{D}=$ VAT 11019, Rs.
Z. $43^{\prime}-50^{\prime}$
Kopie: unten Abb. 7-8, S. 36.

1 A Vs. 1 [ÉN udug ḩul (?) nam-tar $u_{4}$ hu uš-gin 7 an-eden-na mu-un-d $u_{7}-d u_{7}$ ] (Lücke von maximal 2 Zeilen)

$1^{\prime} \quad$ B Vs. $1^{\prime}$

] $\times \times \times x$

$2^{\prime}$ A Vs. 1' [an]š e?-「bil? [

B Vs. 2' $\left[\right.$ a-ḩa-an-gin ${ }_{7}$ b]a-an-ši-ḩa-an

3' [ ki-manu-še]-e un-ni-iš

3' A Vs. 2' [ud]ug ḩul a-lá ḩu[l

B Vs. 4' 'udugl hul a-lá [hul eden-na-t]a é-tùr-ra ba-an-dib

5' ú-tuk-ku lem-nu a-lu-ú lem-nu ina șe-rim tar-ba-șa ib-ta-'u-u

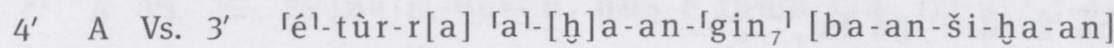

B Vs. 6' é-tùr-ra a-ḩa-an-gin 7 ba-an-ši-hra-an

$7^{\prime} \quad$ tar-ba-șa ki-ma nu-še-e un-ni-iš

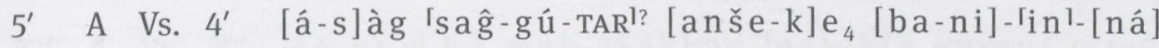

B Vs. 8' 'á-sàg ${ }^{\top}$ sa $\hat{g}-g u ́-T A R$ anše-ke ${ }_{4}$ ba-ni-Iin ${ }^{\top}-n a ́$

$9^{\prime} \quad a^{-}{ }^{\mathrm{I}} s a k^{\top}-k u$ ina ú-re-e si-si-i uš-ni-il-ma

6'f. A Vs. 5'f. [an]še-bi ka-[b]i saḩar-r[a ba]-「an-s[i] / [n]á??-'bil-dè bí-[in-kúr (?)]

B Vs. 10' anše-b[i k]a-bi saḩar-ra ba-an-si ki-tuš-bi-šè ba-an-kúr

$11^{\prime} \quad$ šá $^{\top} i^{1}$-me-re-e šú-nu-ti e-pe-ri pi-i-šú-nu ú-mal-li-ma ru-bu-us-su-nu

$12^{\prime}$ (leer) ú-nak-ki-ir

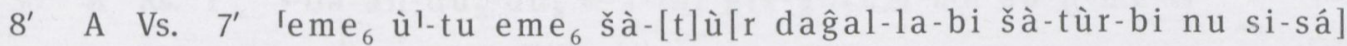

B Vs. 13' ème ù-tu ème šà-tùr daĝal-la-bi šà-tùr-bi nu si-sá

14' a-ta-nu a-lit-tú a-ta-nu mu-rap-pi-iš-tú šá-as-su-ru šá-as-sur-ši-na ul uš-te-šir

15' na-a-ti a-li-da-a-ti na-a-ti šá- (leer) (unterer Rand)

$9^{\prime}$ A Vs. $8^{\prime}$ anše $g a-n a \hat{g} \times{ }^{\prime} \mathrm{ga}^{1}[$ ]

B Rs. 1 dùrùr ga-nâg ga nu-[sub(-)]

2 mu-ur(-)ni-〈is- $\rangle q i$ ši-iz-bi ši-iz-ba ul ú-[šenneqā (?)]

C Vs. $1^{\prime}$ [an] š [e ]

$2^{\prime} \quad$ [AN]ŠE.MES 'Š ${ }^{1}$ ? - $[i z-b i$ ši $]-{ }^{\top} i z^{1}-b[a$

(Strich)

47 Für die Erlaubnis, die vier Keilschrifttexte hier veröffentlichen zu dürfen, sei der Deutschen Orient-Gesellschaft, dem Vorderasiatischen Museum zu Berlin sowie den Trustees des Britischen $\mathrm{Mu}$ seums herzlich gedankt. 
$10^{\prime}$ A Vs. $8^{\prime}$

B Rs. 3 amaš-bi su-mu-ug-ga-ta bí-[in-ĝar]

4 ina su-pu-ri-ši-na i-dir-tu iš-š $[a k-n a]$

C Vs. 3' [an $]_{\text {šd }}$ úr-b[i $\left.\mathrm{s}\right] \mathrm{u}-\mathrm{mu}$-ug-g[a-ta

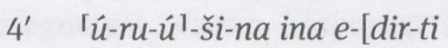

(Strich)

$11^{\prime}$ A Vs. 9' udug $\operatorname{sig}_{5}-\mathrm{g}[\mathrm{a}$

B Rs. 5 [ud]ug sig $_{5}$-ga é-tùr-ra mu-un-[ dib(?)]

6 [še-e]d dum-qí ina tar-ba-și e-ti-i[q]-Гma ${ }^{\top}$

C Vs. 5' rudugl sig $_{5}$-ga é(„KID“)-tùr-ra x [

6' še-e-du dam-qu ALAD tar-ba- ${ }^{[}{ }^{\prime} i^{1} \times$ [ ] ]

(Strich)

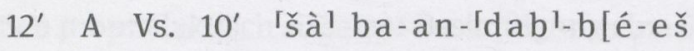

B Rs. 7 [šà ba]-an-dab-bé-eš bar-šè ba-an-gu $u_{4}-u d-d a$

$8 \quad[\mathrm{x}-n]$ u-um-ma ina $a-h a-a-t i$ iš-ta-hi-it

C Vs. $7^{\prime}$ šà ba-an-dab-ba-kám bar-šè b [a?-

$8^{\prime}$ DIĜIR.MEŠ-šú X-nu-I $m a^{1} a-n a a-h a^{\prime}-t i[$ ]

(Strich)

13' A Vs. 11' dAsal-1[ú-ḩi

B Rs. 9 [dAsa]l-lú-ḩi dumu Eriduki-ga-ke ${ }_{4}$

10 [dA]MAR.UTU mar Eri-dù

C Vs. 9' drAsall-lú-ḩi dumu Er[iduki-ga-ke $\left.{ }_{4}\right]$

$10^{\prime}$ dAMAR.UTU DUMU [ ]

(Strich)

14' A Vs. 12'f. ká ĝ gánun-na-ke 4 ] / [ ]

B Rs. 11 [ká ag]run-na-ke ${ }_{4}$ ĝ̀̀ri ĝen-na-ke ${ }_{4}$ tùr-bi igi im-ma-an-sì

12 [ina] ' $b a^{1}$-bi ku-um-mi ina i-tal-lu-ki-šú tar-ba-șu šú-a-tú ip-pa-lis-ma

C Vs. $11^{\prime}$ [k]á agrun-na-ke ${ }_{4}$ gub-b[u-dè] Vs.13' [é]-tùr-ra igi im-[ma-an-sì]

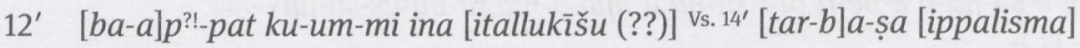
(Strich)

(Strich)

$15^{\prime}$ A Vs. $14^{\prime} \quad$ I

B Rs. 13 [a-a-n]i d+En-ki-ra é-a ba-ši-in-ku ${ }_{4}$ gù mu-un-na-an-dé-e

14 [ana a-b]i-šú d $\hat{E}-a$ ana bé-e-te i-ru-um-ma $i$-šá-as-si

C caret

$16^{\prime}$ A Vs. $15^{\prime}$ |

B Rs. 15 [

$-\mathrm{du}]_{7}$ ?

C Vs. 15' [udug hull (?) nam-t[ar u $]_{4}$ hुuš-gin ${ }_{7}$ ed[e]n-na [ ]

16' [ú-tuk-ku lem-nu (?)] nam-tar (u) [k]i-ma $u_{4}$-mi ez-zi [ina șēri iș̣anundū(?)] (Strich)

$17^{\prime}$ ff. A Vs. $16^{\prime}$

B Rs, 16 [dAsal-lú-hi a-na / níg ĝá]-「el / ĝen-na dumu-ĝu

C Vs. $17^{\prime}$ [dAsal-1]ú-ḩi [ ]

(abgebrochen)

$20^{\prime}$ A Vs. $17^{\prime}$ [

giù]ri-gal šu u-m

18 [ ]'úl-ri-gal-li li-qé-ma 
$21^{\prime}$ A Vs. $18^{\prime}$

B Rs. 19

20

é-tùr-r]a mu-un-taka ${ }_{4}$

] tar-ba-șa pi-te-ma

$22^{\prime}$
A Vs. $19^{\prime}[\mathrm{x}(\mathrm{x})] \mathrm{x} \times \mathrm{x}[$
]- $\mathrm{rbu}$ ?
B Rs. 21
22

23' A Vs. 20' [níĝ-n]a g[i-izi-lá é-tùr-ra(?) ù]-me-ni-è

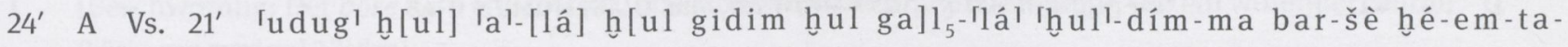
$\mathrm{gub}$

25' A Vs. 22' Idl[udug sig $_{5}$-ga dlamma] sig ${ }_{5}$-ga ḩé-em-da-súg-súg-ge-ě̌

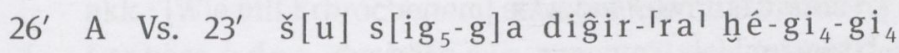

27' A Vs. 24' [dNin-eden-na ĝá-nun (?) s]ikil alim-ma giùri-「gall-bi ḩé-a [zag-ba ḩé]-gub(Strich)

28' A Vs. 25' ka-inim-'rma' [é]-tùr anše-kur-ra sikil-e-da-kám (Strich)

29' A Vs. 26' ÉN [na]m- ${ }^{-} \operatorname{tar}^{1} \mathrm{u}_{4}$ ḩuš-e tumu-gin 7 [ri-a(?)]-àm

$30^{\prime}$ A Vs. 27' $\left[u_{4}\right]$ 'ḩušl?-e kun sù-sù-e an-'edent-na-ke ${ }_{4}$

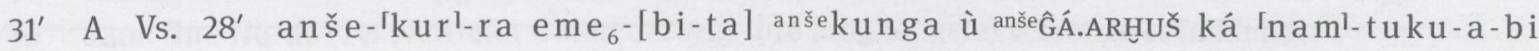

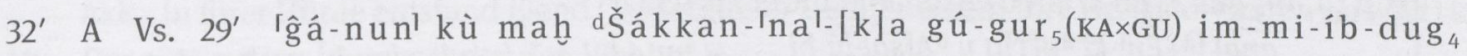

$33^{\prime}$ A Vs. 30' $[\mathrm{x}(\mathrm{x})] \mathrm{x}$ dک̌ákkan ur-saĝ eden-na-ka

34' A Vs. 31' [(Spuren von etwa 4 Zeichen) $] \mathrm{sa} \hat{\mathrm{g}}-\mathrm{ki}$ - bi kúk ku

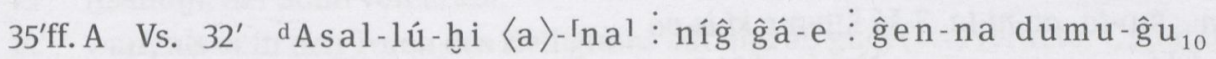
(Rand)

38' A Rs. 1 giba-an-du $u_{8}-d u_{8}$ ĝišá-lal ĝiš-gurum-ma šu ù-me-ti

$39^{\prime}$ A Rs. 2 a-ba $t u_{6}$ kù-za ù-mu-e-ni-sì

$40^{\prime}$ A Rs. $3 \quad \mathrm{tu}_{6} \mathrm{kù-za}$ na ù-me-ni-de $\mathrm{e}_{5}$

41' A Rs. 4 ĝiššinig úin-nu-uš ú(-)sikil-le kur-ra šà-ba ù-me-ni-šub

42' A Rs. 5 níĝ-na gi-izi-lá šu ù-me-ti

43' A Rs. 6 udug ḩul a-lá ḩul gidim ḩul gal 5 -lá huul-dím-ma bar-šè ḩé-em-ta-gub

D Rs. 1' [ú-tuk-ku lem-nu a-lu-ú lem-nu ețemmu lem-nu ga]l-lu-ú le[m-nu ina ahâti]

$2^{\prime} \quad l i-i[z]-{ }^{\top} z i^{1}-[i z]$ (Strich)

44' A Rs. 7 dudug sig $_{5}$-ga dlamma sig ${ }_{5}$-ga ḩé-em-da-súg-súg-ge-eš

D Rs. 3' [dudug sig ${ }_{5}-$ ga d lamm]a sig $_{5}$-ga hyé-en-da-súg-[súg-ge-ě̌] (Doppelstrich) 
45' A Rs. 8 dŠákkan eden ur-sâg-ĝá-ke ${ }_{4}$ gáa-nun kù maḩ ùri-gál-bi hué-a zag-ba ḩé-gub (Strich)

D caret

46' A Rs. 9 ka-inim-ma é-tùr anše-kur-ra sikil-e-da-kám

(Strich)

D caret

47' A Rs. 10 KìD.KìD.BI úIGI-lim úIGI-NIŠ útar-muš Ú-AŠ NUMUN Ú-AŠ

D Rs. 4'f. [ úim-hnu]r-lim úim-hुur-aš-[re] / [útar-muš Ú-AŠ] NUMUN Ú-AŠ

48' A Rs. 11 úSIKIL úel-kul-la úeli(UGU)-kul-la úLÚ.U 19 .LU BAR BAL.GI ${ }^{\mathrm{ku}}{ }^{\prime}$

D Rs. 5'ff. úSIKIL.[LA] / [úel-kul-la] rúleli(UGU)-kul-la úLÚ.U[ ${ }_{19} \mathrm{LU}$ ] / [BAR BAL.GI ${ }^{\mathrm{ku}}$ ]

49' A Rs. 12 BAR Ní̂̂.BÚN.NA er-gi-la hi-lam-mu-ú ka-zi-ra Ĝ́́R.〈TAB〉 BABBAR

C Rs. $1^{\prime}$ [ ] (Spuren) [

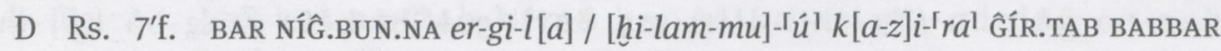

$50^{\prime} \quad \mathrm{A} \quad$ Rs. 13 Ì KU $\mathrm{I}_{6}$ İ ŠAH İ.NUN.NA İ.ĜIŠ ESIR ku-up-ra IM.BABBAR

C Rs. 2' [ ] (Spuren) [ ] ]

D Rs. $8^{\prime}$ f. İ $\mathrm{KU}_{6} /\left[\quad k u-u \mid p-{ }^{\mathrm{I}} r a^{\mathrm{I}}[\right.$

(abgebrochen)

51' A Rs. 14 DIŠ-niš tuš-te-mid ANŠE.KUR.RA.MEŠ ma-la ĜÁL-ú TAG.MEŠ-at

C Rs. $3^{\prime}$ [ ]x ĜÁL-[ ]

(Strich)

52' A Rs. 15 IMIN GI.DU ${ }_{8} a n a^{\mathrm{d}}+E n-k i^{\mathrm{d}}{ }^{\prime}{ }^{\prime}{ }^{\mathrm{d}}$ Asal-lú-ḩi DIĜIR LÚ d15 LÚ

C Rs. 4' [ ana $]^{\left\lceil\mathrm{d}+E n^{\mathrm{l}}-k i^{\mathrm{d}} \mathrm{U}[\mathrm{T}] \mathrm{U} u\right.} \mathrm{d}^{\mathrm{d}} A[$ sal-lú-ḩi

53' A Rs. 16 dNIN-EDEN ù dŠákkan KEŠDA-ma SÍSKUR BAL-qí uzuZAG.LU ME.HÉ

C Rs. 4'f. [ ]/[ ] uzuZAG uzuME.Hु[É]

54' $\mathrm{A}$ Rs. 17 ù KA.NE ĜAR-an ŠID-ta $a_{5} a n-n i-t a_{5}$ 3-šú ŠID-ma ki-is-pa

C Rs. 5'f. [ ]/[ క̌] $\mathrm{ID}^{-}{ }^{-} m a^{1} k i-i s-p a$

55' A Rs. 18 ana GIDIM IM.RI.A-šú ta-ka-sa-ap gišUTUG.UD.MEŠ ŠUB- $d i$

C Rs. 6'f. 'ana' G[IDIM ]/[ ] ŠUB

56' A Rs. 19 giÙRI.GAL ta-za-qip i+na UB gišUTUG.UD PÚ te-hye-er-ri

C Rs. $7^{\prime}$ ina KÁ $\left.{ }^{\top}\right|_{E} ^{\prime}()[$.

57' A Rs. 20 mí+EŠGAR tu-ka-sa-ma ana ŠÀ PÚ ŠUB-di i+na UGU PÚ ĜIS.MEŠ tu-pa-rak

C Rs. $8^{\prime}$ [ ina muhhi bür-t]im ĜIŠ.ÙR.MEŠ t $t$ [u-pa-rak]

58' A Rs. 21 'EĜTIR-ki-ta ${ }_{5}$ ANŠE.KUR.RA.MEŠ $i+n a$ UGU $t u$-še-taq 1 M[Áš] ina EN.NU.UN.USAN(,DUR+RI“)

C Rs. 9'f. [ ] ina muh-hi [ ]/[ ]

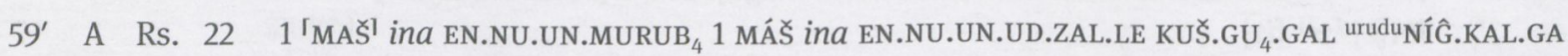

C Rs. $10^{\prime}$ [ ] ] [

(abgebrochen)

60' A Rs. 23 has-ḩal-la-ta qu-ta-ri tuš-ba-’i-šu-nu-ti 
61' A Rs. 24 A.MEŠ A.GÚB.BA tu-lal-šu-nu-ti

(Strich; dann Leerraum)

Kol. A Rs. 25 GABA.RI ${ }^{\hat{i} i s ̌ l} l e-` i$ SAR KUR UR $\left[\mathrm{I}^{\mathrm{k}}\right]^{\mathrm{i}}$ LIBIR.RA ana KA LIBIR.RA-šu

26 IRi-ba+a-tu DUMU Ri-še-i[a ]]úMAŠ.MAŠ MAN IN.SAR

(Leerraum; kurz vor dem unteren Rand abgebrochen)

\section{Übersetzung}

1 [Beschwörung: Der böse udug (?),48 der Todesbote, wirbelt ${ }^{49}$ durch die Steppe wie ein wütender Sturm]. (Lücke von maximal 2 Zeilen)

$1^{\prime} \quad[$

$2^{\prime} \quad$ sum.: [Wie mit Erbrochenem besudelte er] deren [Pfe]rde. akk.: [Wie mit Erbrochenem] schwächte er [die Pferde].

3' Der böse u d ug, der böse a la, machte ${ }^{50}$ sich auf von der Steppe zum Viehhof.

$4^{\prime}$ sum.: Wie mit Erbrochenem besudelte er den Viehhof. akk.: Wie mit Erbrochenem schwächte er den Viehhof.

5' Der a s a g (-Dämon) ließ an den Stellplätzen der Pferde (die Tiere) am Boden lagern ${ }^{51}$,

$6^{\prime}$ die Mäuler jener Hengste füllte er mit Erde und

$7^{\prime}$ sum.: [feindete (die Tiere) an], während sie dalagen (so Textvertreter A; Textvertreter B statt dessen: feindete (die Tiere) an ihrem Lagerplatz an). akk.: machte ihre Lagerplätze lebensfeindlich.

8' Den fohlenden Stuten und den trächtigen Stuten ${ }^{2}$ brachte er den Mutterleib nicht in Ordnung.

$9^{\prime}$ Die Milch trinkenden Fohlen [können sie] nicht [stillen $]^{53}$ mit Milch.

10' sum: Ihre Hürde (Textvertreter C statt dessen: Ihre Junghengste) versetzte er in Elend. akk.: In ihrer Hürde entstand Elend (Textvertreter C statt dessen: Ihre Hengste? [waren versetzt] in E[lend]).

11' Der gute udug [durchschritt] den Viehhof, 54 akk. (Textvertreter C): Der gute Geist, der Geist der Viehhürde, [ging vorbei],

$12^{\prime}$ sie brachten ihn in Zorn ${ }^{55}$, und da sprang er zur Seite.

13' Asalluhi, der Sohn von Eridu,

$14^{\prime}$ warf, als er in den Toren des Heiligtums auf und ab ging (Textvertreter C statt dessen: stand), ein Auge auf jenen Viehhof, 56

15 trat zu seinem Vater Enki/Ea ein in das Haus und rief ihm dabei zu:

16' „[Der böse udug], der Todesbote, wirbelt durch die Steppe wie ein wütender Sturm.“

$17^{\prime}$ „[Asalluhi, was (ist es, das du nicht weißt)? Was soll ich dir noch beibringen]?

$18^{\prime} \quad$ [Alles, was ich] (weiß, das weißt auch du)!

$19^{\prime}$ Geh hin, mein Sohn, (Asalluhi),

$20^{\prime}$ [ ] nimm [(Zahl?)] Standarten,

21' [ ] öffne (?!) den Viehhof,

22' [ ] richte [die (Zahl?) Standarten] auf,

23' beschwenke [den Viehhof(?)] mit [Räucher]gefäß und Fa[ckel].

24' [Der böse udug, der böse ala, der böse Totengeist, der] Böses anrichtende galla möge dann zur Seite treten!

25' [Der gute u dug], die gute [la m m a] mögen ihm (d.h. dem Eigner des Viehhofs) zur Seite stehen!

26' [Den guten Händen] des Gottes sei er (d.h. der Eigner des Viehhofs)] anvertraut!

48 In der Übersetzung sind diejenigen Wörter oder Passagen kursiv gesetzt, deren Ergänzung als unsicher zu gelten hat.

49 In der nicht erhaltenen akkadischen Fassung stand wohl statt dessen: [(sie) wirbeln hin und her].

50 In der akkadischen Fassung: (sie) machten sich auf.

51 In der akkadischen Fassung ist hinzugefügt: und dann.
52 In der ersten akkadischen Fassung des Textvertreters B statt dessen: Der fohlenden Stute und der trächtigen Stute etc.

53 Oder: Die Milch trinkenden Fohlen [können] Milch nicht [saugen]. 54 In der akkadischen Fassung ist hinzugefügt: und dann.

55 Textvertreter $\mathrm{C}$ und die akkadische Fassung anders.

56 In der akkadischen Fassung ist hinzugefügt: und dann. 
27' [N i n-e d e n - n a (?)] sei doch die Standarte für die [rei]ne, majestätische [Wohnung (?)]! Sie möge fest an dem vorgesehenen Platz stehen!“

Wortlaut der Beschwörung, (die dafür bestimmt ist,) einen Pferdehof zu reinigen.

Beschwörung: Der Todesbote, der wütende Sturm, der [weht] wie der Wind, der [wütende Sturm], der mit dem Stachel löckt, der aus der Steppe (kommt), hat die Pferde [und deren] Stuten, die Maultiere und die (zugehörigen) Maultierstuten, deren Mutterleib keinen Ausgang hat, in der heiligen, erhabenen Wohnung des Schakkan niedergemacht. Da war [das] ... des Schakkan, des Helden der Steppe, [ (und auch)] seine Stirn verfinstert. „Asalluhi, was (ist es, das du nicht weißt? Was soll ich dir noch beibringen)? Alles, was ich (weiß, das weißt auch du)! Geh hin, mein Sohn, (Asalluhi), nimm einen Schöpfeimer mit (hölzernem) Henkel, gib deine heilige Beschwörung über jenes Wasser (und) mache es mit deiner heiligen Beschwörung rein. Wirf Tamariske (und) Seifenkraut, das reine Kraut des Gebirges, dort hinein. Nimm Räuchergefäß und Fackel.

Der böse u dug, der böse a la, der böse Totengeist, der Böses anrichtende galla möge dann zur Seite treten! Der gute u dug, die gute la m m a mögen ihm (d.h. dem Eigner des Viehhofs) zur Seite stehen!

Schakkan, der (Gott) von Steppe und Gebirge!?, sei doch die Standarte für die heilige, erhabene Wohnung! Er möge fest an dem vorgesehenen Platz stehen!

Wortlaut der Beschwörung (die dafür bestimmt ist), einen Pferdehof zu reinigen.

Die zugehörigen Ausführungen: Du verarbeitest „Es-stellte-sich-Tausend-entgegen-Kraut“, „Es-stellte-sich-Zwanzig-entgegen-Kraut“, Lupine, šammu ēdu-Kraut, Samen des šammu ēdu-Krautes,

sikillu-Pflanze, elkulla-Pflanze, elikulla-Pflanze, amīlānu-Pflanze, Panzer? einer raqqu-Schildkröte, Panzer? einer šeleppû-Schildkröte, Wanderheuschrecke, hilammu-Heuschrecke, kazīru-Wurm, weißen Skorpion, Fischtran, Schweinefett, Butterschmalz, (pflanzliches) Öl, Bitumen, Asphalt (und) Gips zu einer homogenen Masse. Alle Pferde, die da sind, rührst du damit an.

Sieben Tragaltärchen richtest du für Enki, Schamasch, Asalluhi, ${ }^{57}$ den Gott des (betroffenen) Menschen, ${ }^{58}$ die Göttin des (betroffenen) Menschen,

$3^{\prime}$ die Herrin-der-Steppe und Schakkan auf. Dann bringst du ein (Schlacht)opfer dar. Schulterfleisch, Fettgewebe 4'f. und Bratfleisch legst du (ihnen) hin. Diese Beschwörung rezitierst du dreimal. Dann bringst du dem Totengeist seiner Familie ${ }^{59}$ das Totenopfer dar. Du errichtest Rohrhütten.

6' Du richtest eine Standarte (Textvertreter C zusätzlich: im Tor der Vieh[hürde]) auf. In einer Ecke (jeweils) einer Hütte gräbst du eine Grube.

Du bindest ein weibliches Zicklein und wirfst es dann in die Grube hinein. Die Grube überdeckst du mit Holzbalken. Daraufhin läßt du die Pferde darüber schreiten. Du beschwenkst sie mit einem Ziegenbock in der Nachtwache der Abenddämmerung,

$9^{\prime}$ mit einem (weiteren) Ziegenbock in der mittleren Nachtwache, mit einem (weiteren) Ziegenbock in der Nachtwache des frühen Morgens, mit einer mit einem Rinderfell bespannten Pauke, mit einer Glocke?,

57 Textvertrerter C: Schamasch und Asalluhi. 58 D.h. für den Gott des Menschen, dem der Pferdehof untersteht oder gehört.
59 Gemeint ist der Totengeist des Menschen, dem der Pferdehof untersteht oder gehört. 
60 mit Blatt- (und) Räucherwerk.

61' Du reinigst sie mit Wasser aus dem Weihwassergefäß.

Kol. Die Vorlage war eine hölzerne Tafel, ein altes Schriftstück aus dem Land Akkad. Entsprechend dem Wortlaut des alten Originals schrieb (dies) Ribatu, der Sohn des Rischeja, der Beschwörer des Königs.

\section{Kommentar}

1 Die erste, leider in keinem Textvertreter erhaltene Zeile kann nach Z. 16' ergänzt werden; denn der Struktur jener Beschwörungen entsprechend, die A. Falkenstein in LSS NF 1, 44-76 als den ,Marduk-Ea-Typ“ beschrieb, ist in dem vorliegenden Text die in der ersten Zeile zusammengefaßte Unheilsbeschreibung an späterer Stelle noch einmal wiederholt (hier Z. 16'). Dort ist sie dem Asalluhi/Marduk in den Mund gelegt als ein an den göttlichen Vater Enki/Ea gerichteten Bericht über das den Menschen angetane Übel, gegen das die Beschwörung Hilfe verspricht. Die Ergänzung der ersten Zeile darf auch deshalb als sicher gelten, da in dem ansonsten zweisprachig gehaltenen Textvertreter B in Z. 16' auf eine akkadische Interlinearübersetzung verzichtet wurde. Dies geschah in den assyrisch geschriebenen Bilinguen aus Koujunjik nämlich in der Regel nur dann, wenn die entsprechende Interlinearübersetzung bereits zuvor an anderer Stelle niedergeschrieben worden war. Die nicht erhaltene akkadische Fassung der ersten Zeile (vgl. dazu Textvertreter C, Vs. 16') muß gelautet haben: utukku lemnu (?) namtar(u) kīma ümi ezzi ina șēri ișșanundū.

$1^{\prime}$ Die in Textvertreter B erhaltenen Zeichenspuren gehören zu der akkadischen Interlinearübersetzung. Die Verbalform am Zeilenende läßt sich vorerst nicht rekonstruieren.

2' Die in Textvertreter A erhaltenen Spuren sind so geringfügig, daß die Ergänzung des Zeilenanfangs unsicher bleibt. Die weiteren Ergänzungen richten sich nach Z. 4'. Wie in Z. $4^{\prime}$ ist in der akkadischen Fassung a- ha -

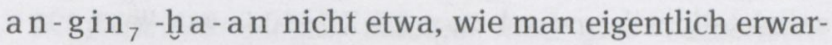
ten würde, mit der Wendung kīma nušê nešû, „wie mit Erbrochenem besudeln", wiedergegeben worden, sondern mit kīma nušê unnušu (enēšu D), „wie mit Erbrochenem schwächen“. Hier muß freilich gegen CAD N/II 193a s.v. nešû nicht zwangsläufig ein Irrtum vorliegen. Der Übersetzer des Textes mag durchaus absichtlich mit dem Gleichklang der Wurzeln nešû und enēšu gespielt haben, um mit hermeneutisch-,etymologischen“ Mitteln zu zeigen, daß die in der Beschwörung beschriebene Kontamination (kīma nušê nešû) zu der Schwächung (unnušu) der Betroffenen führt. - Dem Text liegt wohl die (möglicherweise auf konkreten Erfahrungen beruhende) Vorstellung zugrunde, daß die „durch die Steppe wirbelnden“ Dämonen zunächst die (wild lebenden) Tiere der Steppe mit Krankheit belegen (Z. $\left.2^{\prime}\right)$, um erst dann in den menschlichen Lebensraum vorzudringen (Z. $3^{\prime}$ ) und den Viehhof und die domestizierten Tiere anzugreifen (Z. 4 'ff.). Das -bi

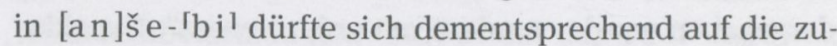
vor genannte Steppe beziehen.

4' Siehe den Kommentar zu Z. 2'.

$5^{\prime}$ an š e, die sumerische Bezeichnung für die Equiden, wird im Akkadischen sonst regelmäßig mit imēru, „Esel“, wiedergegeben. Die an dieser Stelle vorliegende Gleichung mit sisû, „Pferd“, ist einzigartig und scheint dadurch motiviert, daß in der akkadischen Übersetzung ein uralter Text, der aus Zeiten stammt, als Pferde in Mesopotamien noch keine nennenswerte Verbreitung gefunden hatten, den Gegenwartsbedingungen der mittelassyrischen Zeit angeglichen wurde.

$6^{\prime}$ an š e = imēru, „Esel“, ist hier in der Bedeutung „Hengst“, „männlicher Equide“ gebraucht.

$7^{\prime}$ Die Ergänzung von Text A Vs. 6' muß angesichts des sehr schlechten Erhaltungszustandes der Tafel unsicher bleiben. In sumerisch-akkadischen Bilinguen ist die Entsprechung ná = rubșu bezeugt (siehe CAD R 395a).

$8^{\prime}$ è me / e $\mathrm{me}_{6}=$ atānu, „Eselin“, ist hier in der Bedeutung „Stute“, „weiblicher Equide“ gebraucht. Da es gegen alle Schreiberkonventionen verstieße, wenn $n a-a-t i$ tatsächlich - wie offenbar in den Wörterbüchern angenommen (siehe AHw 86b; CAD A/II 481b, CAD Š/II 145b) eine abgekürzte Schreibung für atānāti wäre, muß in nâti eine unregelmäßige Pluralform zu atānu gesehen werden. Das in Textvertreter B, Vs. $15^{\prime}$ folgende šá ist nur verständlich, wenn man es als Abkürzung für das in der vorangehenden Zeile vollständig ausgeschriebene šá-as-sur-ši-na ul uš-te-šir auffaßt. Demzufolge muß nâti ein AkkusativObjekt sein.

$9^{\prime} \mathrm{Zu}$ dieser Zeile vgl. die im CAD an verschiedenen Stellen (CAD M/II 215f. s.v. mūr nisqi; CAD N/II 33 s.v. nașābu und CAD Š/III 149a s.v. šizbu) vorgeschlagene Ergänzung: ga nu-[sub] = šizba ul u[nașșab].60 Gegen diese Ergänzung der akkadischen Zeile spricht freilich die in Textvertreter B, Rs. 4 folgende akkadische Interlinear-

60 Statt $u[$ nașșab] würde man wohl eher $u[$ nașșabū ] erwarten. 
übersetzung, in der sich das Suffix -šina auf die in Z. $8^{\prime}$ genannten Stuten beziehen muß. Der gesamte akkadische Text wäre erheblich harmonischer, wenn „die Stuten“ Subjekt der akkadischen Fassung der Zeile 9' wären. Aus diesem Grunde wird hier die Ergänzung ušenneqā bevorzugt. Für die Verbindung g a - s ub (KA×GA) ist die akkadische Entsprechung enēqu Š-Stamm bezeugt (siehe CAD E, 165a).

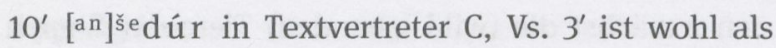
phonetische Schreibung für d ù r ùr (so in Textvertreter B, Rs. 1) aufzufassen, obgleich es nicht mit mūr nisqi, mūrnisqu oder mūru, „Fohlen“, „,erlesenes Jungtier“ wiedergegeben wurde, sondern mit urû, „Hengst“. Die akkadische Fassung von Textvertreter B legt allerdings nahe, daß in C, Vs. 4' überhaupt nicht von Pferden die Rede ist, sondern wie in Z. $5^{\prime}$ von dem ebenfalls urû geheißenen Stall (AHw. urû $(m)$ I; CAD U urû A).

$11^{\prime}$ In Textvertreter A stand im Zeilenanfang, anders als in A, Vs. $22^{\prime}$ und Rs. 7, nicht d ${ }^{\mathrm{d}} \mathrm{dug} \mathrm{sig}_{5}$ - $\mathrm{g}$ a sondern lediglich udug $\operatorname{sig}_{5}$-ga. In Textvertreter $C$ ist das Zeichen é - so wie bereits aus der Kopie CTN 4, Nr. 101 hervorgeht - mit drei (anstelle von vier) stehenden Keilen geschrieben. Dennoch steht in Textvertreter C, Vs. $5^{\prime}$ nicht, so wie es C, Vs. $6^{\prime}$ eigentlich nahelegt, alad tù r-ra oder la mma tùr-ra. In C, Vs. $5^{\prime}$ ist vielleicht é-tùr-ra-š [è zu lesen.

$12^{\prime}$ In Z. 12' dürfte das sumerische Verb šà da b vorliegen, auch wenn es nicht gelingen will, die sumerischen mit den stark voneinander abweichenden akkadischen Fassungen dieser Zeile in Einklang zu bringen. š à $\mathrm{d} a \mathrm{~b}$ wird in den sumerisch-akkadischen Bilinguen sonst mit akkadischem zenû, „zürnen“, wiedergegeben (siehe AHw 1519 und CAD Z 85). Es bleibt freilich völlig unklar, aufgrund welcher Überlegungen šà $b a-a n-d a b-b e ́-e \breve{~}$ bzw. šà ba-an-dab-ba-kám mit [x-n]u-um-ma bzw. DIĜIR.MEŠ-šú $\mathrm{x}-n u^{-}{ }^{-} m a^{1}$ wiedergegeben wurde. Eine Form von zenû kann hier keinesfalls vorliegen, und es gelingt nicht, für die akkadischen Zeilen einen überzeugenden Ergänzungsvorschlag zu machen. DIĜIR.MEŠ-šú in Textvertreter C, Vs. 8' hätte nur dann in der vorangehenden sumerischen Zeile eine Entsprechung, wenn der Übersetzer das-an-in šà ba-an-dab-ba-kám als Schreibung für das Wort „Gott“" (di g i i r) gedeutet hätte. Wäre dem so, hätte der Übersetzer damit wohl einen Beweis für sein fehlendes Verständnis des Sumerischen geliefert. Die Weise, wie die Spatien in Textvertreter C, Vs. $7^{\prime}$ verteilt sind, legt in der Tat nahe, daß der vermutlich noch junge Schreiber der Tafel unfähig war, eine korrekte Analyse des sumerischen Textes zu liefern. Denn zwischen šà ba-an$\mathrm{d} a \mathrm{~b}-\mathrm{ba}$ - und dem zweifellos zugehörigen -kám ist ein großer Freiraum gelassen, während das -kám so eng an das folgende bar-š è gérückt ist, daß der Eindruck entsteht, der Schreiber habe in der Zeichenfolge KÁM BAR KU eine einzige syntaktische Einheit gesehen.

$14^{\prime}$ Es ist nicht ganz auszuschließen, daß - wie die Textpartitur von Z. 14' nahelegt - in Textvertreter A die Zeichenfolge GÁ NUN als Variante zu der Schreibung É.NUN aufzufassen und a gru $\mathrm{n}_{\mathrm{x}}$ zu lesen ist. In diesem

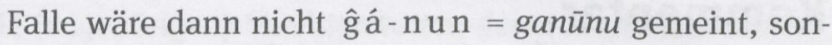
dern wie in den Duplikaten a grun = kummu. Gleiches könnte auch für das ĝ á-n u n in Text A, Vs. 29' (Z. 32') und Rs. 8 (Z. 45') gelten.

$16^{\prime}$ In Textvertreter B fehlt die akkadische Interlinearübersetzung, da diese bereits in der nicht erhaltenen ersten Zeile des Textes stand und so an dieser Stelle nicht wiederholt wurde. In Textvertreter $\mathrm{C}$ ist im Anfang der Zeile Vs. $16^{\prime}$ für die hier erwartete Ergänzung [ú-tuk-ku lem-nu (?)] möglicherweise etwas zu wenig Platz. Im Anfang der Zeile Vs. $15^{\prime}$ verlangen die Raumverhältnisse jedoch die Ergänzung von zwei Zeichen.

$17^{\prime}-19^{\prime}$ Vgl. die Parallelstelle unten Z. $35^{\prime}-37^{\prime}$. Zu dieser Passage, der sogenannten Marduk-Ea-Formel, vgl. neben A. Falkenstein, LSS NF 1 53-58 z. B. Šurpu V-VI 31-35 und CT 17, 26: 60-63 (jeweils mit akkadischer Interlinearübersetzung) sowie zahllose Parallelen aus der āšipūtu-Literatur. Die vollständigen Zeilen lauten:

${ }^{d} A s a l-l u ́-h i$ a-na nu-ì-zu a-na a-ra-abta hy-e = Marduk mīnâ lā tīde mīnâ luraddīka

ní ĝ gá-e ì-zu-a-ĝ u ${ }_{10}$ ù za-e in-ga-e-zu =ša anāku ìdû attā tīde

\section{Marduk}

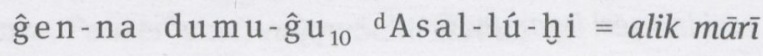

$21^{\prime}$ In der sumerischen Fassung findet sich, anders als in der akkadischen, wider Erwarten keine imperativische Verbalform. In der ersten Zeilenhälfte könnte von der Hürde $($ a maš $=$ supūru) die Rede gewesen sein, vielleicht in einer weitgehend parallelen Formulierung.

22' Für eine mögliche Ergänzung der Zeile ist die Parallelstelle udug-h hul-a-meš XII 160 (= BIN 2, 33: 194 = Geller, SAACT 5,165) zu Rate zu ziehen: giurì-gal sa ĝĝa-na mu-un-da-gub-gub-bu = MIN ina re-ši-šú ú-zaq-qip.

23' Die Ergänzung richtet sich nach udug-hula-meš XIII-XV 176 (= Geller, SAACT 5, 173): nî ĝ-na gi izi-lá u-me-ni-è = níg-na-ku GI.IzI.LÁ šu-bi-i’-šú-ma. Siehe auch u dug-ḩul-a-meš VII 13 (= Geller, ebd. 136) und XVI 90 (= Geller, ebd. 182).

$24^{\prime}-25^{\prime}$ Die Zeilen $24^{\prime}-25^{\prime}$ wurden nach der Parallelstelle Z. $43^{\prime}-44^{\prime}$ ergänzt.

$25^{\prime}$ Vgl. die Parallelstelle udug-ḩul-a-meš II 79 (= M. Geller, SAACT 5, 100): [udug sig $_{5}-$ g] a d la mma 
sig $_{5}$-ga hé-en-súg-súg-ge-eš mit der recht freien akkadischen Übersetzung: še-e-du dum-qi la-mas-si dum-qí i-da-a-šú lu-ú ka-a-a-an sowie ferner u d u g- hu u la-meš V 195 (= Geller, ebd. 127); XIII-XV 143 (= Geller, ebd. 172), 181 (= Geller, ebd. 173), 222' (= Geller, ebd. 175) etc.

26' Die sehr häufig in der sumerischen Beschwörungsliteratur belegte Zeile wurde folgendermaßen ins Akkadische übertragen: ana qātī damqāti ša ilīšu lippaqid.

$27^{\prime}$ Z. $27^{\prime}$ stellt eine genaue Parallele zu Z. 45' dar und wurde entsprechend ergänzt. Wie in Z. 45' war im Anfang der Z. 27' eine Gottheit genannt, die einer Räumlichkeit „Standarte“ sein möge. Manches spricht dafür, daß hier die „Herrin-der-Steppe“ (Nin-eden-na = dBēlet-șêri(NINEDEN)) genannt war, die laut Z. 53' neben dem göttlichen Richtergremium und den persönlichen Göttern des Besitzers der zu schützenden Tiere gemeinsam mit Schakkan als Schutzgottheit der Pferde beopfert wurde. Daher ist es recht wahrscheinlich, daß in dem hier beschriebenen Ritual sowohl dBēlet-sẹeri (Z. 27') als auch Schakkan (Z. 45') in Gestalt von Standarten den Pferden Schutz bieten sollten. Zu Z. $27^{\prime}$ vgl. auch die Parallelstelle STT 198: 29 (mīs pî):

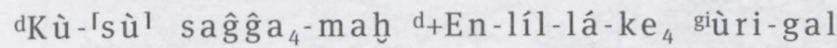

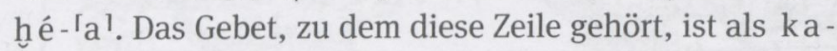
inim-ma gi ùri-gal-a-kám (STT 198: 33) bezeichnet und wurde über eine aufzurichtende Standarte rezitiert, die wie dieStandarten von Nin-e den-na und Schakkan Schutz bieten sollte. Das Zeichen ALIM hat in Textvertreter A, Vs. 24' (statt GÌr×A.LIM) die Form Gìr.A.LIM.

$29^{\prime}$ Die Ergänzung des Zeilenendes richtet sich nach dem sumerischen Sprichwort: nam-tar $\mathrm{u}_{4}$ hुuš kala m-ma ri-a, „Das Schicksal ist ein wütender Sturm, der über das Land einher braust“ (siehe B. Alster, Proverbs of ancient Sumer I, 48 Sammlung 2.13). Vgl. ferner u d u g hyul-a-meš XVI 44 (= Geller, SAACT 5, 179): an šàga-ta tumu-gin 7 kalam-ta zi-ga-meš $=i \check{\text { - }}$-tu qéreb AN-e ki-ma šá-a-ri ana ma-a-ti it-te-bu-ni šú-nu.

31' Der Textlogik zufolge muß das sumerische anšêิÁ.ARHUSU eine Bezeichnung für das dem Maulesel (anšekunga = parû) zugehörige weibliche Tier sein. Die Aussprache des mit der Zeichenfolge anšêิÁ.ARHuS geschriebenen sumerischen Wortes ist unbekannt (die für ARHUS belegte Lesung e $\mathrm{m} \mathrm{e}_{8}$ [siehe Borger MesZL ${ }^{2}$ S. 479] erinnert freilich an das sumerische Wort für Eselin è $\mathrm{me}=$ atānu). Im Akkadischen wird das weibliche Maultier atān parî genannt (siehe W. G. Lambert, BWL, 218:15).

32' Die Ergänzung des Zeilenanfangs richtet sich nach der Parallelstelle Z. 45'. Zu g á - n u n in Textvertreter A siehe den Kommentar zu Z. 14'. Zu dem Gott Schakkan/ Sumuqan siehe A. Cavigneaux/F. N. H. Al-Rawi, A scho- lar's library in Meturan?, in: T. Abusch/K. van der Toorn (Hg.), Mesopotamian magic. Textual, historical, and interpretative perspectives (Groningen 1999) 251-273, besonders $261 \mathrm{ff}$. sowie F. Pomponio, OrNS 53 (1984) $1 \mathrm{ff}$.

$33^{\prime} \mathrm{Im}$ Anfang der Zeile ist vielleicht: [s a $\mathrm{g}$ ] $-{ }^{[} \mathrm{ki}^{1} \mathrm{zu}$ lesen.

$35^{\prime}-37^{\prime}$ Vor der Zeile Textvertreter A, Vs. $32^{\prime}$ ist ein hauchdünner Strich zu sehen.

$38^{\prime}$ In Text A, Rs. 1 steht - wie zu erwarten - im Original tatsächlich giba - und nicht wie in KAR 91 gis u - . Vgl. die Parallelstelle CT 17, 26: 64: Igišlba- an- $d u_{8}-d u_{8}$ (á-)lal-e ĝiš-gurum-ma šu u-me-ti mit den Interlinearübersetzungen: MIN-e ĜIS kip-pa-ti li-qé-ma; mIN-e a-lal(!)-le-e iș-și kip-pa-tum li-qé-e. Siehe auch F. A. M. Wiggermann, Mesopotamian protective spirits (Groningen 1992) 66 mit weiteren Parallelstellen.

39' Vgl. die Parallelstelle CT 17, 26: 67: a-bi t $\mathrm{u}_{6}$ k ù $\mathrm{za}-\mathrm{na} \mathrm{u}$-me-ni-sì mit der Interlinearübersetzung: ana A.MEŠ šú-nu-ti ši-pat-ka KÙ-ti i-di-ma.

40' Vgl. die Parallelstelle CT 17, 26: 68: $\mathrm{tu}_{6}$ kù-za $\mathrm{na}$ u-me-ni-d ${ }_{5}$ mit der Interlinearübersetzung: ina tee-ka el-li ul-lil-ma.

41' Es bleibt unklar, ob man in ú sikil(-le) ku r-ra, der „reinen Pflanze aus dem Gebirge“ - ähnlich wie in der Lugalbanda-Erzählung I, Z. 307, 319 und 337 (úi-li-in-nu-uš ú sikil kur-ra-ka; siehe ETCSL 1.8.2.1) ein Attribut zu der zuvor genannten Pflanze sehen sollte, oder ob in Z. 41' unseres Textes der Name eines dritten, dem Wasser hinzuzufügenden Krautes úsikil $=s i-$ killu (siehe AHw. 1041a und CAD S 243f.) genannt ist. Laut Uruanna I 19 (siehe CAD S, 243b), wo ú - si kil als ú maltakal ša šadî, „Seifenkraut des Gebirges“, bezeichnet wird, ist ú-sikil freilich eine nur im Gebirge aufzufindende Unterart des Seifenkrautes. Eben dieses Kraut sikillu wird auch in Z. $48^{\prime}$ als Ingredienz der Salbe genannt, die zum Schutz von Pferden eingesetzt werden sollte. Auch wenn die Schreiber in Z. 41' unseres Textes gewiß die Lesung ú(-)sikil-le kur-ra im Sinne hatten, wäre die Form ú(-)sikil-le grammatisch nicht zu erklären. Sollte daher nicht doch ursprünglich ŝiššinig úin-nu-uš ú-sikil li kur-ra gemeint gewesen sein? li stünde dann wohl für ŝ̀iš (ši - $) l i \mathrm{i}=$ burāšu ,Wacholder“.

42' Es ist eigenartig, daß die Anwendung von Räucherbecken und Fackel (so wie oben in Z. 23') hier streng genommen nicht eigens genannt ist. Sollte hier eine Zeile fehlen?

43'-44' Vgl. die Parallelstelle Z. 24'-25'.

$45^{\prime}$ Vgl. Z. 32' und zu der zweiten Zeilenhälfte die Parallelstelle Z. 27'. Im Zeilenanfang dürfte ur-sa ĝ fehlerhaft für h hur - s a g stehen. In der sumerischen Literatur ist Schakkan, der Gott der Tiere, jedenfalls mehrfach als 


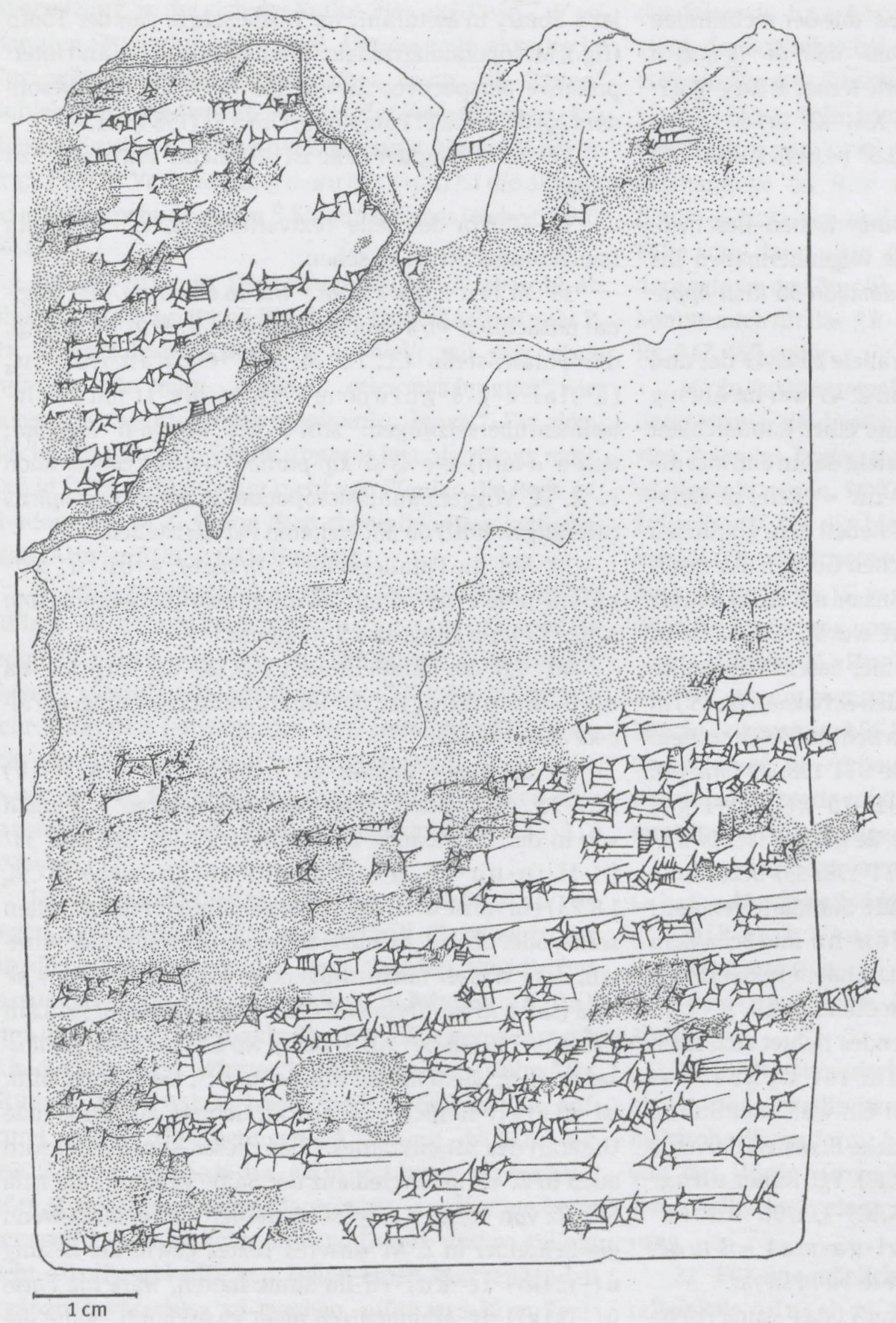

Abb. 1: VAT 10035 Vs. (Ms. A) 


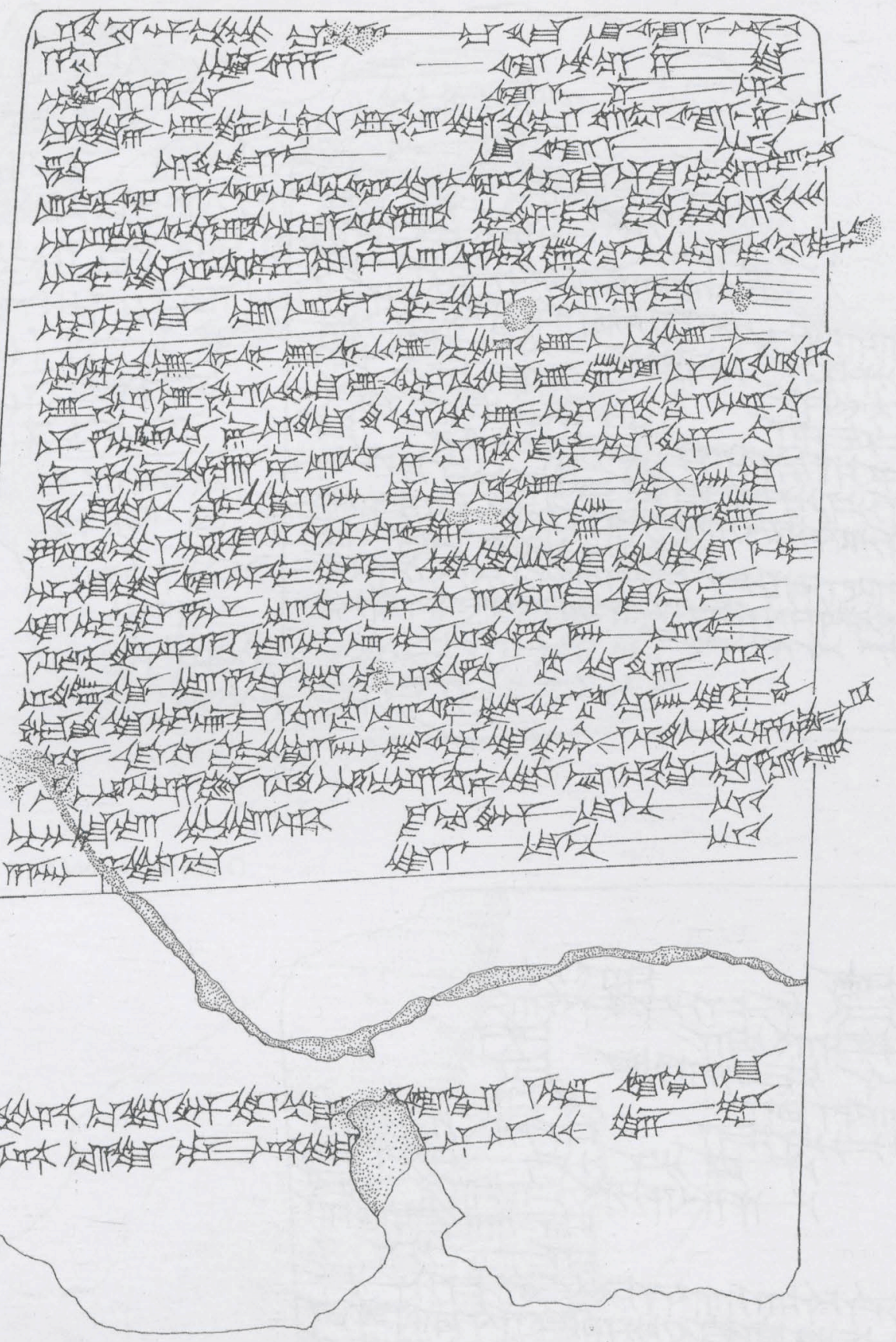

Abb. 2: VAT 10035 Rs. (Ms. A) 


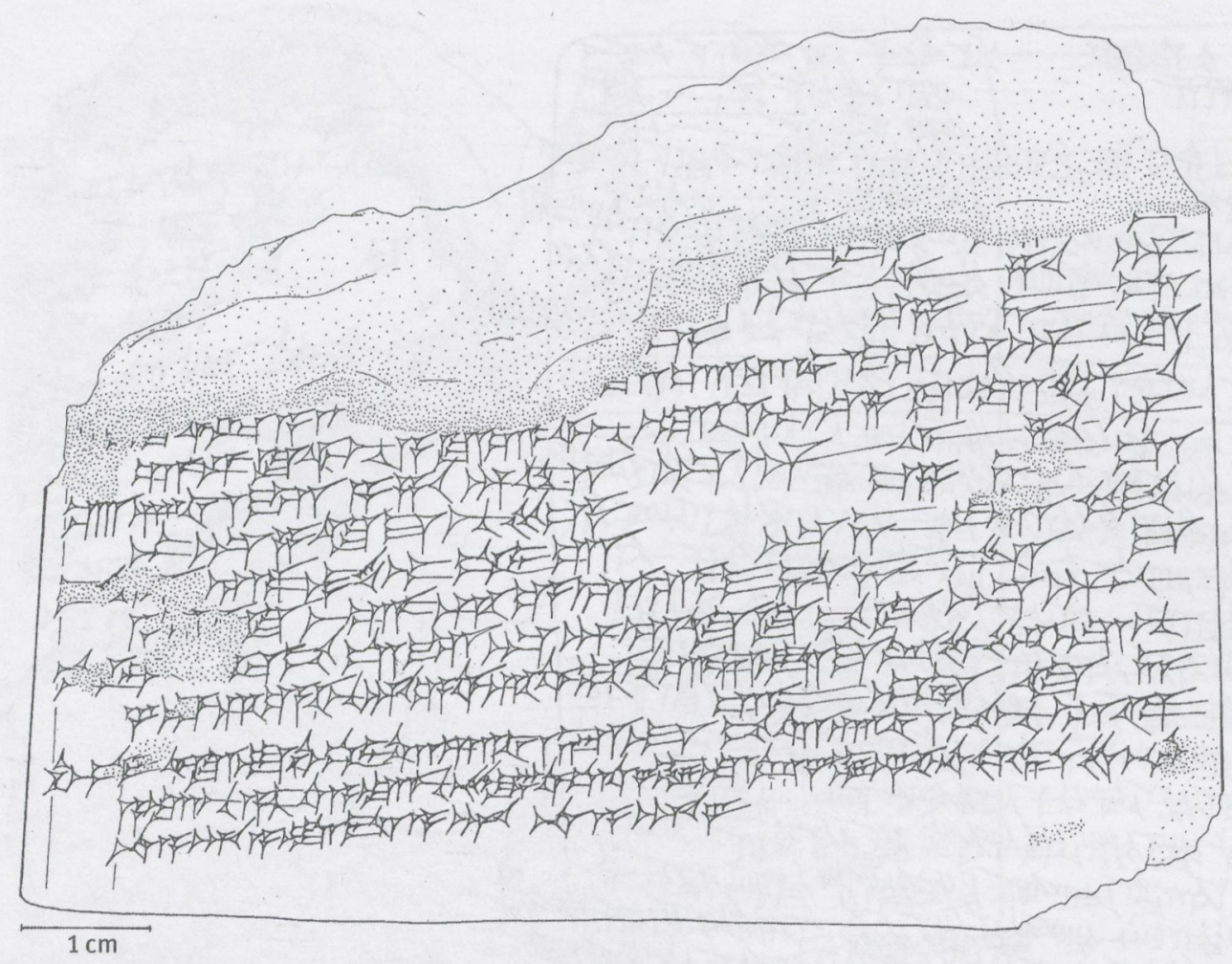

Abb. 3: Sm 1708 Vs. (Ms. B)

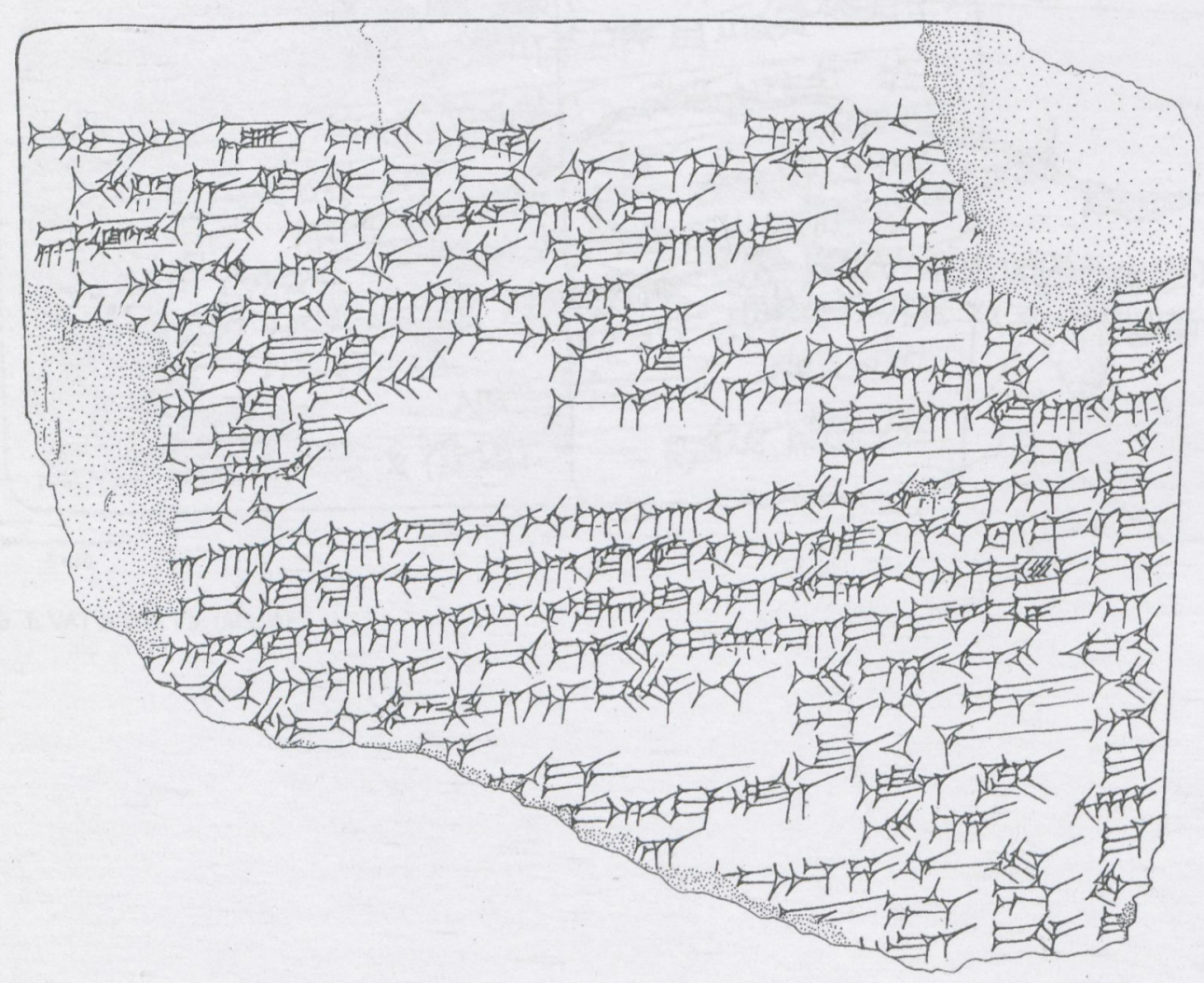

Abb. 4: Sm 1708 Rs. (Ms. B) 


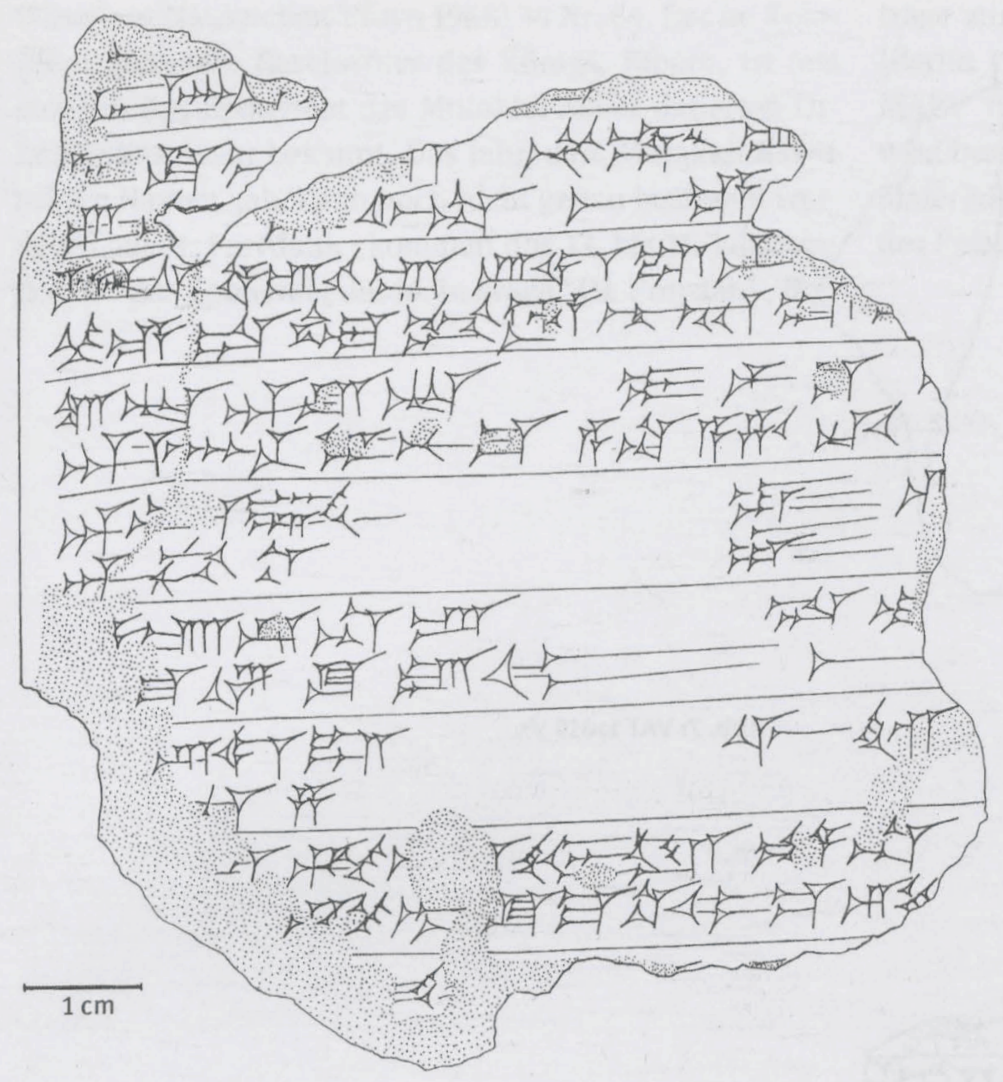

Abb. 5: ND 4405/47 Vs. (Ms. C)

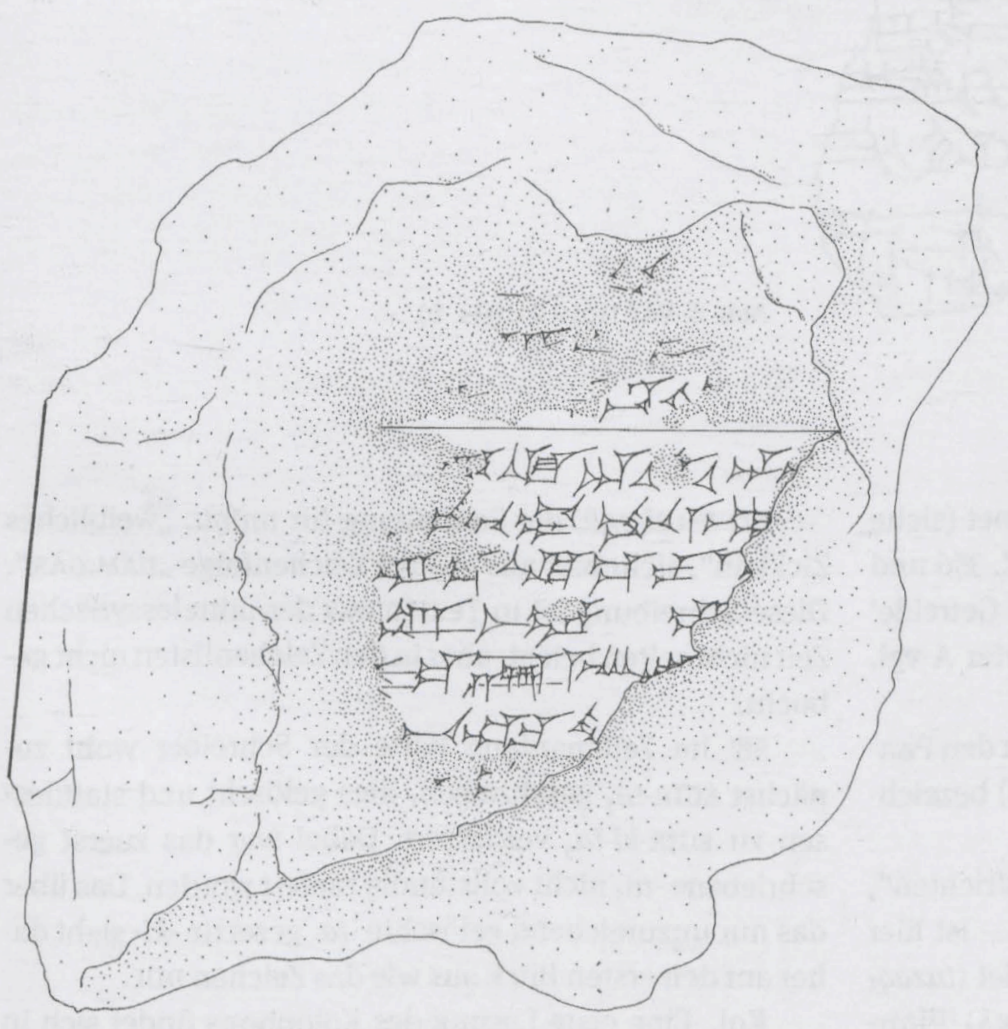

Abb. 6: ND 4405/47 Rs. (Ms. C) 


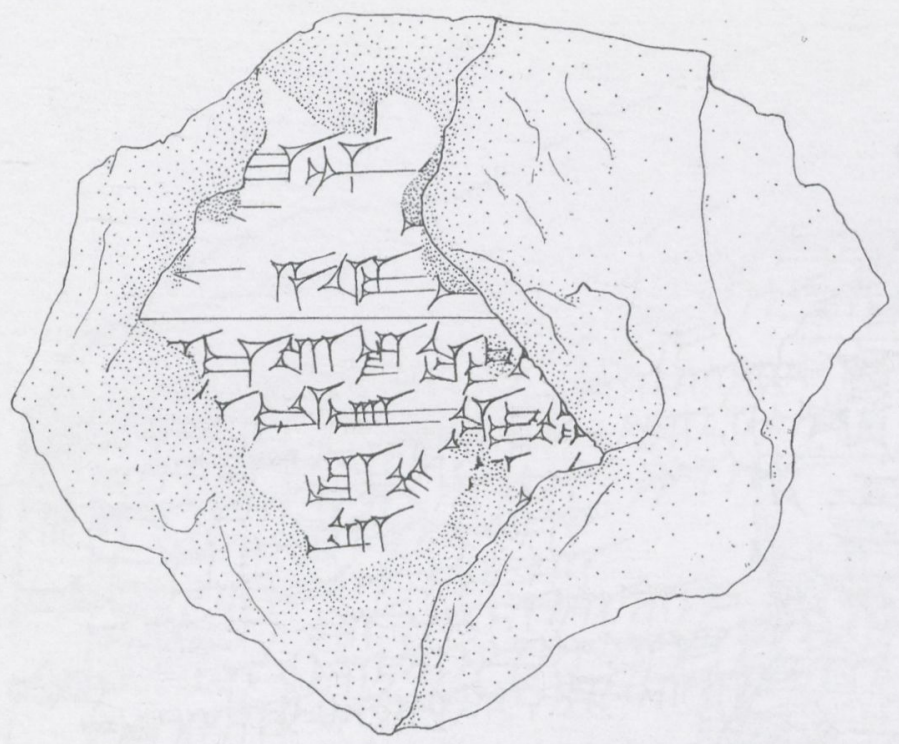

Abb. 7: VAT 11019 Vs.

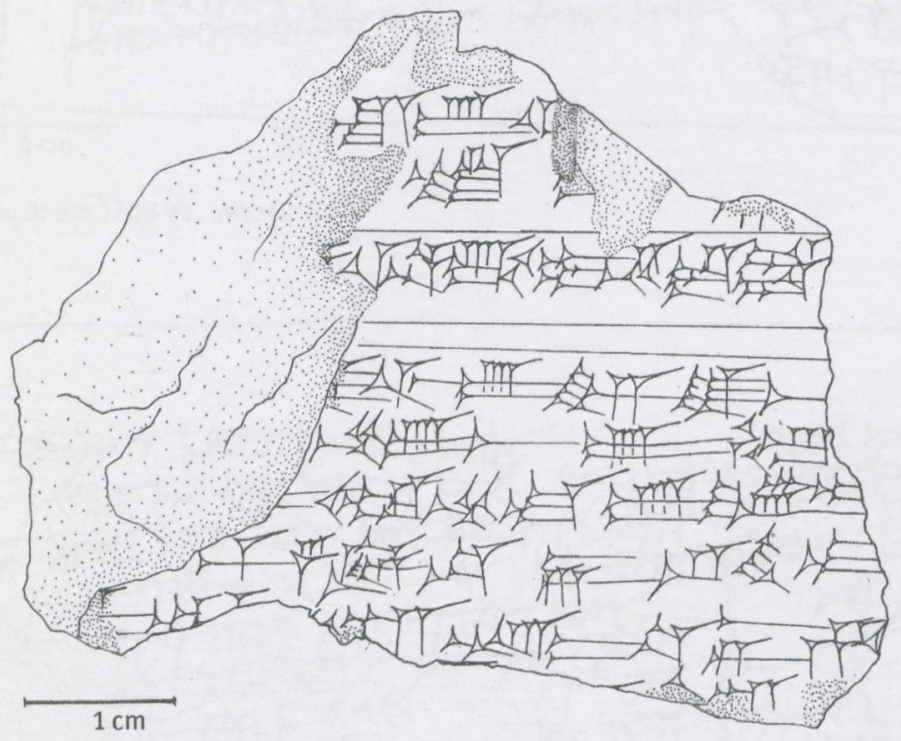

Abb. 8: VAT 11019 Rs. (Ms. D)

dŠákkan lugal huur-sa ĝ- ĝá-ke ${ }_{4}$ bezeichnet (siehe z.B. ,Enki und die Weltordnung ' [ETCSL 1.1.3], Z. 356 und das ,Streitgespräch zwischen Mutterschaf und Getreide“ [ETCSL 5.3.2], Z. 97). Zu ĝá-nun in Textvertreter A vgl. den Kommentar zu Z. 14'.

48'f. Mit CAD Q 297a dürfte quleptu hier eher den Panzer als die Haut der Schildkröten (so AHw 926b) bezeichnen.

56' Das Präsens des Verbs zaqāpu, „aufrichten“, „pflanzen“ - sonst der Ablautklasse zugehörig - ist hier wie das Präsens eines Verbs der i-Klasse gebildet (tazaqqip). In Textvertreter C, Rs. $7^{\prime}$ ist wohl ina bāb(KÁ) rél[tarbași(TÙR) zu lesen. $57^{\prime}$ mí +EŠGAR, die Schreibung für unīqu, „weibliches Zicklein“, sieht hier aus wie die Zeichenfolge „DAM.GÀR“. Diese Schreibung ist in Texten aus der mittelassyrischen Zeit nicht selten belegt, aber in den Zeichenlisten nicht gebucht.

$58^{\prime}$ Im Zeilenanfang hatte der Schreiber wohl zunächst EGIR- $t a_{5}$ geschrieben, dies gelöscht und stattdessen zu EGIR-ki-ta $a_{5}$ verbessert. Dabei war das zuerst geschriebene $-t a_{5}$ nicht vollständig radiert worden. Das über das nur unzureichend gelöschte $-t a_{5}$ gesetzte $-k i$ - sieht daher auf den ersten Blick aus wie das Zeichen DuL.

Kol. Eine erste Lesung des Kolophons findet sich in H. Hunger, Babylonisch-assyrische Kolophone. AOAT 2 
(Kevelaer/Neukirchen-Vluyn 1968) 34 Nr. 64. Der im Kolophon genannte Beschwörer des Königs, Rībātu, ist aus einer in das Eponymat des Mutakkil-Aššur datierten Urkunde aus Assur bekannt. Das Jahr, dem Mutakkil-Aššur seinen Namen gab, kann noch nicht genau bestimmt werden. Laut H. Freydank „kommen das 13. bis 11. Jahrhundert für das Eponymat des M. in Frage“ (H. Freydank, Bei- träge zur mittelassyrischen Chronologie und Geschichte [Berlin 1991] 154). In dem von D. Prechel/H. Freydank, MARV 10, 23 veröffentlichten Schriftstück (VAT 10195) wird bescheinigt, daß der Beschwörer ('úmAŠ.MAŠ) Rībātu Materialien entgegengenommen hatte, die „für das Ritual des Palastes (ana nēpeše ša ekalli)“ benötigt wurden. 